\title{
El uso de animales por las sociedades agropastoriles tempranas: análisis zooarqueológico de un basural de la aldea de Palo Blanco (valle de Fiambalá, Catamarca)
}

(1) Juan P. Miyano*

Recibido: 15 de marzo de 2017

Aceptado:

6 de junio de 2017

\section{Resumen}

En este trabajo se presenta el análisis del conjunto arqueofaunístico proveniente de un basural de la aldea de Palo Blanco (valle de Fiambalá, Catamarca). Específicamente, se identifican cambios y continuidades en las estrategias vinculadas a la obtención, manejo, aprovechamiento y consumo de animales desarrolladas por las tempranas sociedades agropastoriles de la aldea. La explotación de camélidos domésticos se registra desde los inicios de la ocupación de la aldea (ca. 400 a.C.) y se combina con el aprovechamiento de fauna silvestre pequeña en los momentos más tempranos (400 a.C. - 400 d.C.) y con la explotación de vicuñas en los momentos más tardíos (post 400 d.C.). Principalmente, las llamas se criaron para el consumo de carne, médula ósea y grasa aunque también hay evidencia que sugiere su uso como animales de carga, productoras de fibra e incluso el empleo de sus huesos para la manufactura de instrumentos. El desarrollo y consolidación de la estrategia pastoril habría sido posible en un contexto aldeano en el cual la agricultura aportaba los recursos principales para el sostenimiento de la población mientras que la caza otorgaba recursos complementarios.

Animal use in early agro-pastoral societies: zooarchaeological analysis of a midden from the village of Palo Blanco (Fiambalá Valley, Catamarca)

\footnotetext{
Abstract

In this article, we present the archaeofaunal analysis of an assemblage recovered from a midden located in the village of Palo Blanco (Fiambalá Valley, Catamarca). This analysis allowed us to identify changes and continuities among Palo Blanco's earliest agro-pastoral societies in regard to animal procurement, management, exploitation, and consumption strategies. The exploitation of domestic camelids was recorded from the beginning of the occupation of Palo Blanco (ca. $400 \mathrm{BC}$ ). During the earliest period (400 BC - A.D. 400), domestic camelid use was combined with the exploitation of

* Instituto de las Culturas (IDECU), Museo Etnográfico “J. B. Ambrosetti”, Facultad de Filosofía y Letras, Universidad de Buenos Aires (UBA) - CONICET. Moreno 350 (CP C1091AAH) Ciudad Autónoma de Buenos Aires, Argentina. E-mail: jpmiyano@gmail.com
}

\section{Palabras Clave}

Zooarqueología Camélidos Pastoreo Valle Catamarca Formativo

\section{Keywords}

Zooarchaeology Camelids Herding Valley Catamarca Formative 
small wild animals, while after A.D. 400 it was supplemented by the hunting of wild vicuñas. Llamas were raised mainly for their meat, bone marrow and fat; however, these domestic camelids were also used as beasts of burden and as a source of fiber to make textiles and bone to manufacture artifacts. The development and consolidation of this pastoralist strategy was possible in a village context where agriculture provided the main source of sustenance for the villagers while hunting provided additional resources.

\section{Introducción}

En este trabajo se reportan los resultados obtenidos del análisis de los conjuntos arqueofaunísticos provenientes de la excavación de un basural situado en la localidad arqueológica de Palo Blanco. Esta localidad se emplaza $1 \mathrm{~km}$ al oriente del pueblo homónimo, en el valle de Fiambalá (departamento de Tinogasta, Catamarca). Presenta un total de seis núcleos habitacionales $(\mathrm{NH})$ los cuales se distribuyen de manera dispersa. De acuerdo con los fechados radiocarbónicos realizados en los distintos $\mathrm{NH}$, la aldea de Palo Blanco habría estado ocupada por aproximadamente 800 años, entre los siglos III y X d.C. (Miyano, De Nigris y Ratto, 2015). Sin embargo, nuevos fechados realizados sobre el material proveniente de la excavación del basural indican el uso de este espacio en el período comprendido por los siglos IV a.C. y IV d.C. Estos nuevos datos cronológicos le otorgan mayor profundidad temporal a la aldea, coincidiendo con el inicio del modo de vida productivo en los valles mesotermales del noroeste argentino (Olivera, 2001).

El objetivo principal es abordar las prácticas humanas vinculadas a la obtención, manejo, aprovechamiento y consumo de animales durante los momentos más tempranos de ocupación hasta ahora registrados en la aldea de Palo Blanco y, para ello, se estudian los conjuntos arqueofaunísticos recuperados del basural. Específicamente, se analizan los cambios y/o continuidades en el uso de los animales en tres momentos temporales diferentes (400 a.C. a 1 d.C., 1 d.C. a 400 d.C. y post 400 d.C.) definidos arbitrariamente a partir de los fechados radiocarbónicos obtenidos de las capas estratigráficas naturales. Así, se evalúa el rol de la caza y el pastoreo y la importancia relativa de los camélidos y su uso con fines nutricionales y tecnológicos en la aldea a través del tiempo. La evidencia zooarqueológica permite ampliar el conocimiento acerca del uso de los animales por parte de las sociedades agropastoriles tempranas de la aldea de Palo Blanco, así como también otorgar información para la región valliserrana del noroeste argentino.

\section{Antecedentes}

\section{La localidad arqueológica de Palo Blanco}

La localidad arqueológica de Palo Blanco se emplaza en el sector norte del valle de Fiambalá $\left(27^{\circ} 20^{\prime} 17.9^{\prime \prime} \mathrm{S} 67^{\circ} 44^{\prime} 33.3^{\prime \prime} \mathrm{O}\right)$ a $1900 \mathrm{~m}$ s.n.m. Este valle se encuentra limitado por las sierras de Fiambalá, la cordillera de San Buenaventura y las sierras de Las Planchadas y Narváez cubriendo un área de aproximadamente $1.400 \mathrm{~km}^{2}$ (Figura 1A). En la actualidad, las precipitaciones ocurren principalmente durante el período estival, con un promedio de $300 \mathrm{~mm}$ por año. Las precipitaciones que se desarrollan en las sierras linderas se traducen en cursos fluviales que acarrean sedimento hacia las zonas más bajas del valle. Los vientos son fuertes y provienen fundamentalmente del sudeste, canalizados por las formaciones montañosas contiguas. Tanto la acción fluvial como la eólica contribuyen al proceso de sedimentación del valle y, consecuentemente, al entierro de los sitios arqueológicos allí emplazados (Bonomo, Osella y Ratto, 2010). 

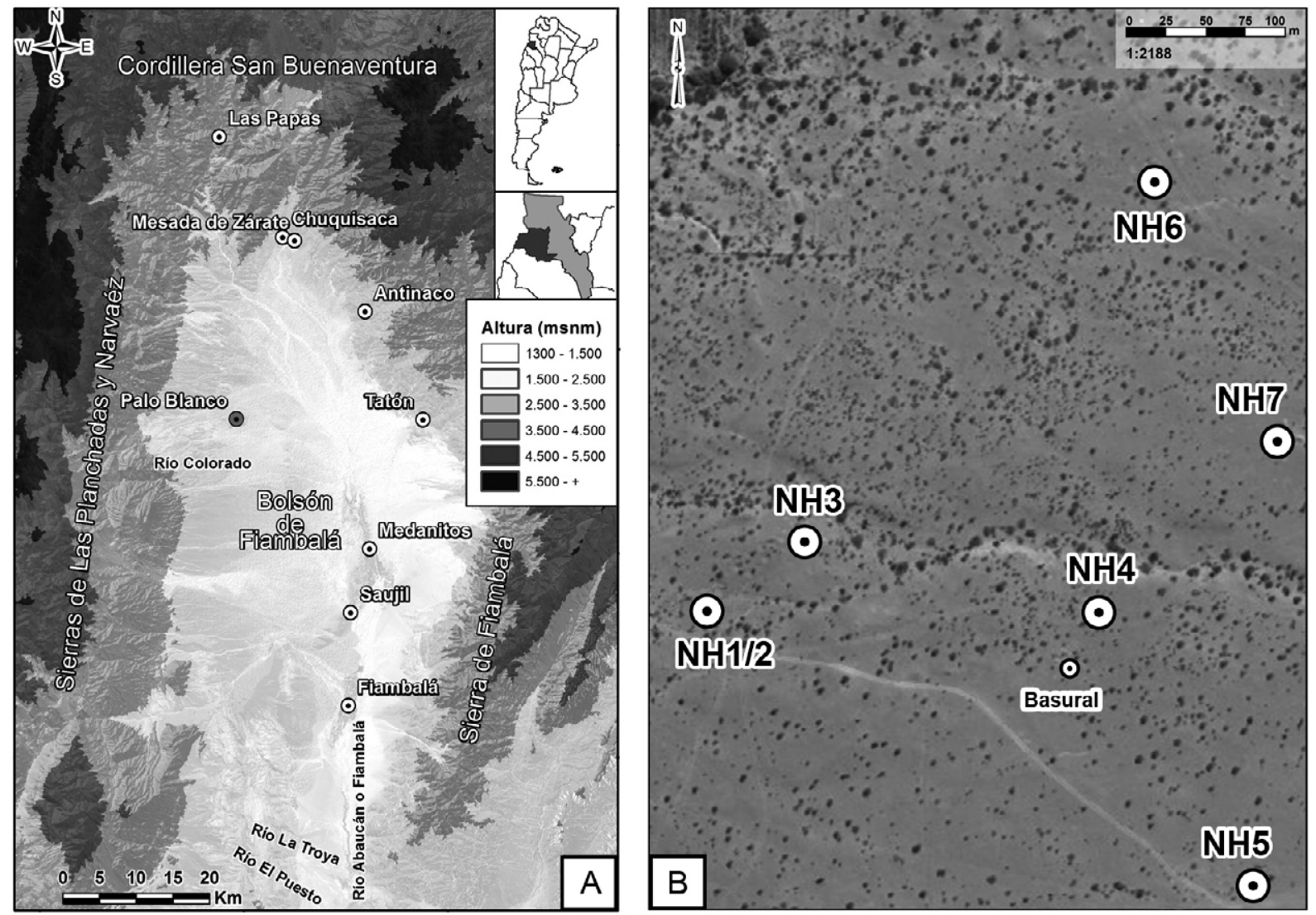

Figura 1. A) Ubicación de la localidad arqueológica de Palo Blanco. B) Distribución de los seis núcleos habitacionales y del basural.

Con respecto a la vegetación, el valle se inserta en la Provincia Fitogeográfica del Monte (Cabrera y Willink, 1973). La formación dominante es la estepa arbustiva la cual se compone principalmente de jarilla (Larrea cuneifolia), brea (Cercidium australe), usillo (Tricomaria usillo), retamo (Bulnesia retama), matasebo (Monttea aphylla) y monte negro (Bougainvillea spinosa). A diferencia de lo que se observa actualmente, los bosques de algarrobos (Prosopis flexuosa), acompañados por atamisqui (Capparis atamisquea) y chañar (Geoffroea decorticans), habrían tenido una gran extensión en épocas prehispánicas (Vervoorst, 1951).

Las primeras investigaciones arqueológicas en la localidad de Palo Blanco fueron realizadas por Sempé (1976), quien registró cinco conjuntos de estructuras asociadas a las que denominó "núcleos habitacionales" y las identificó como NH1, NH2, NH3, NH4 y NH5. Posteriormente, y tras décadas de ausencia de estudios arqueológicos en el área, las investigaciones fueron retomadas en la década de 2000 por el Proyecto Arqueológico Chaschuil-Abaucán. Dada la nula visibilidad y obstrusividad de las estructuras, se recurrió a métodos geofísicos para su relocalización y, potencialmente, la identificación de nuevas estructuras (Ratto y Basile, 2010). La aplicación de estos métodos permitió determinar que dos de los NH identificados por Sempé (1976) (NH1 y NH2) formaban parte de una misma unidad arquitectónica (Osella, Bonomo y Ratto, 2009). Además, se relocalizó y excavó el NH3 (Martino, Bonomo, Lescano, Osella y Ratto, 2006) y se identificaron y excavaron dos nuevas estructuras: el NH6 (Bonomo et al., 2010) y el NH7 (Ratto, comunicación personal, 2017). Así, la localidad arqueológica de Palo Blanco se compone de seis NH de distribución dispersa (Figura 1B) los cuales poseen similitudes en cuanto a su técnica constructiva y al modo de organizar el espacio doméstico: fueron construidos con muros de tapia a partir de la técnica de encofrado, 
presentan forma rectangular y se componen de una cantidad variable de recintos que se conectan entre sí y/o con espacios más amplios (posiblemente patios) (Ratto y Basile, 2010; Sempé, 1976). Respecto a la temporalidad de su ocupación, los NH no fueron habitados simultáneamente sino que lo hicieron de manera alternada durante gran parte del primer milenio de la era (ca. 200-1.000 d.C.) (Miyano et al., 2015).

\section{Uso de animales por las sociedades agropastoriles tempranas en la región valliserrana}

Entre los 1.000 años a.C. y los 500 años a.C. se observa en el registro arqueológico regional del noroeste argentino la aparición de indicadores que sugieren el paso de un modo de vida cazador-recolector a uno productivo (Olivera, 2001). Las principales características de estas primeras sociedades productivas son: a) la incorporación de prácticas agrícolas y/o pastoriles, complementadas con actividades de caza y recolección; b) el desarrollo de un sistema de asentamiento con alto grado de sedentarismo y aparición de aldeas de ocupación permanente; c) la introducción y desarrollo de tecnologías tales como cerámica, metalurgia y textil; d) la constitución de las unidades domésticas como unidades productivas; y e) una organización social relativamente igualitaria con baja jerarquización política (Albeck, 2000; Olivera, 2001, 2012; Ratto et al., 2015). Estas características son rasgos generales y, por lo tanto, pueden variar de región a región. Las estrategias de subsistencia y movilidad se encuentran sujetas a la ecología de la región y a las características culturales de cada grupo humano. Así, y en términos generales, en los valles bajos la agricultura fue la actividad a partir de la cual se organizó el sistema de asentamiento y subsistencia mientras que en la puna ese lugar lo ocupó el pastoreo (Olivera, 2001). Al respecto, las primeras sociedades productivas de la región valliserrana del noroeste argentino desarrollaron una economía mixta, donde la agricultura y el pastoreo eran complementadas con prácticas de cazarecolección (Albeck, 2000).

La permanencia y desarrollo en el tiempo de estas sociedades ("formativas" sensu Olivera, 2001) también varía de región a región. En este sentido, por ejemplo, en el valle de Ambato, la organización social, política y económica característica de estas sociedades resulta reconfigurada hacia el siglo V d.C., a partir del cual se observa un mayor grado de complejidad política y desigualdad social relacionado al período de Integración Regional (Laguens, 2006). Sin embargo, estos cambios no se observan en otros valles del territorio catamarqueño para el mismo momento del tiempo. Por un lado, en el valle de Santa María, las sociedades formativas se extendieron hasta aproximadamente el siglo X d.C., momento en el que comenzaron a desarrollarse los asentamientos aglomerados, localizados sobre mesetas y con acceso restringido característicos del período de Desarrollos Regionales (Scattolin, 2007). Por otro lado, en el valle de Fiambalá y todo el oeste tinogasteño, las sociedades formativas perduraron más allá del primer milenio de la era, evidenciándose una continuidad y repetitividad de las prácticas entre los siglos I y XIII d.C. (Ratto et al., 2015). De este modo, la persistencia en el tiempo de este tipo sociedades es sumamente variable en regiones relativamente próximas.

La zooarqueología de sitios de las primeras sociedades agropastoriles ha tenido un desarrollo relativamente reciente para la región valliserrana. La mayor parte de las investigaciones provienen de sitios del sur de los valles Calchaquíes fechados entre los siglos I a.C. y X d.C. Entres ellos se destacan Loma Alta E y G, Tesoro II, Yutopián, Bañado Viejo (Izeta, 2007), Soria 2 (Belotti López de Medina, 2011), Cardonal (Scattolin et al., 2007), Mesada de Agua Salada (Lanzelotti y Spano, 2015) y Morro del Fraile (Nastri, Coll Moritán y Belotti López de Medina, 2012). Además, para el valle de Fiambalá, recientemente se analizaron los conjuntos arqueofaunísticos provenientes del NH3 y NH6 de Palo Blanco, fechados entre los siglos V y X d.C. (Miyano et al., 2015). 
Con respecto a los sitios del sur de los valles Calchaquíes, los conjuntos arqueofaunísticos allí recuperados se caracterizan por presentar un claro predominio de camélidos. En este sentido, Izeta $(2007,2008)$ analiza las especies de camélidos representadas, la fauna menor y su importancia relativa. Por un lado, observa que la proporción de camélidos domésticos (Lama glama) durante el primer milenio de la era es similar a la de los camélidos silvestres (Lama guanicoe y Vicugna vicugna). Por otro lado, destaca que hacia el final del primer milenio de la era se incrementa la diversidad taxonómica en el registro debido a la mayor abundancia de vertebrados pequeños. Esta presencia de camélidos silvestres y fauna menor sugiere no solo que la caza continuó teniendo importancia durante momentos en que el modo de vida productivo se encontraba ya desarrollado sino que también indica que las llamas (si bien fueron importantes como fuente de alimento dentro de los sistemas aldeanos) no fueron criadas exclusivamente con fines nutricionales (Izeta, 2007). Además, la presencia de Vicugna vicugna indica la relación de estos sitios de la región valliserrana con la puna, hábitat natural de este camélido silvestre (Laker, Baldo, Arzamendia y Yacobaccio, 2006).

Otra tendencia destacada por Izeta $(2007,2008)$ es el mayor aprovechamiento de camélidos adultos con respecto a los subadultos durante el primer milenio de la era, el cual aumenta aún más hacia el final de dicho período. Así, en los sitios del sur de los valles Calchaquíes se conservaron camélidos domésticos hasta una edad adulta (lo cual habría sido posible por el aporte de los recursos provenientes de la agricultura) con el objetivo de aprovechar sus recursos secundarios: fibra y capacidad de carga (Izeta, 2007). Respecto a lo último, otros tipos de evidencias apoyan el aprovechamiento de las llamas adultas como animales de carga y productoras de fibra. Por un lado, en Loma Alta se detectaron dos falanges segundas de Lama glama con patologías óseas, las cuales se relacionan a la sobrecarga de las extremidades durante el desarrollo de actividades de caravaneo (Izeta y Cortés, 2006). Por otro lado, en distintos sitios de la región (Cardonal, Yutopián, Bañado Viejo y Loma Alta) se registraron instrumentos óseos manufacturados sobre metapodios de camélidos que fueron interpretados como parte del toolkit para el desarrollo de la actividad textil (Izeta, Cattáneo, Scattolin y Cortés, 2013). La presencia de estos instrumentos resultan indicadores indirectos que apoyan la idea del desarrollo de prácticas vinculadas a la manufactura de textiles (Izeta, 2007) y, complementariamente, dan cuenta del aprovechamiento de los huesos de los camélidos para la manufactura de artefactos.

Es necesario destacar que no todos los sitios de esta región exhiben las tendencias desarrolladas anteriormente. Por ejemplo, el sitio Soria 2, uno de los sitios más tempranos del valle (ca.100 a.C. - 300 d.C.), presenta diferencias en cuanto a la relación entre camélidos silvestres y domésticos, como así también en la relación entre camélidos adultos y subadultos. En primer lugar, se destaca una preponderancia de camélidos silvestres y, en segundo lugar, predominan los camélidos subadultos. Belotti López de Medina (2011) sostiene que la predominancia de camélidos subadultos en Soria 2 puede responder a diversas causas. Sin embargo, propone que la matanza selectiva de animales subadultos en un sistema ganadero poco desarrollado y con rebaños pequeños es la más plausible considerando la temprana cronología de Soria 2. Esta explicación fue propuesta originalmente por Olivera (1997) para los sitios agropastoriles más tempranos de la puna de Antofagasta de la Sierra.

Finalmente, el análisis arqueofaunístico realizado en el NH3 y NH6 de Palo Blanco otorgó los primeros resultados acerca del uso de animales por las primeras sociedades agropastoriles del valle de Fiambalá (Miyano et al., 2015). Al igual que en los valles Calchaquíes, se observó una clara predominancia de camélidos, identificándose tanto especies domésticas (Lama glama) como silvestres (Vicugna vicugna y posiblemente Lama guanicoe), siendo la fauna menor muy escasa. Al igual que lo resaltado para Soria 2, tanto en el NH3 como en el NH6 se observa una presencia mayoritaria de camélidos 
subadultos lo que sugiere una estrategia de pastoreo vinculada al aprovechamiento de recursos primarios. A pesar de las diferencias temporales en la ocupación del NH3 (ca.500-650 d.C.) y del NH6 (ca. 700-1000 d.C.) no se registran grandes cambios en cuanto al uso de los animales en la aldea de Palo Blanco para la segunda mitad del primer milenio de la era.

En suma, en todos los sitios de sociedades agropastoriles tempranas reseñadas anteriormente, los camélidos se constituyen como el taxón principal. Además, se destaca la presencia de llamas así como también de animales silvestres (principalmente vicuñas y guanacos), lo que sugiere que la caza complementó a las actividades pastoriles. Respecto a las llamas, se emplearon distintas estrategias en cuanto al manejo de los rebaños: en algunos casos se priorizó la producción de recursos primarios (carne, médula ósea y grasa) y en otros la producción de recursos secundarios (fibra y/o capacidad de carga).

\section{El basural de la aldea de Palo Blanco}

En sus investigaciones realizadas en Palo Blanco, Sempé (1976) identificó un basural emplazado a unos $20 \mathrm{~m}$ al sur del NH4, al cual denominó "Círculo de Piedra B". Allí excavó una trinchera de 6 por $2 \mathrm{~m}$ y recuperó distintos tipos de materiales entre los que destacan fragmentos de cerámica tipo Saujil y Ciénaga, restos óseos de camélidos, restos vegetales quemados (marlos de maíz y semillas de chañar y algarrobo), instrumentos y lascas de obsidiana y carbón. Además, señaló que en los alrededores de la trinchera se podían observar "huaqueos".

En la actualidad, la visibilidad de dicho basural resulta nula. Hacia el sur del NH4 se observan excavaciones asistemáticas ("huaqueos"), evidenciadas por depresiones en la superficie del terreno y por la presencia de distintos tipos de materiales arqueológicos asociados a ellas. Se planteó y excavó un sondeo de 2 x $2 \mathrm{~m}$ en un sector aledaño a estas excavaciones que no habría estado sujeto a la remoción de sedimentos. Durante la excavación no se observó ninguna evidencia en la estratigrafía que indique algún tipo de alteración antrópica del pasado reciente. De este modo, se asumió que la disposición y asociación de los materiales arqueológicos recuperados es el resultado de procesos de depositación en los que no tuvieron incidencia ni las excavaciones asistemáticas mencionadas anteriormente ni las realizadas por Sempé durante sus investigaciones.

El sondeo en el basural alcanzó una profundidad de 1,95 m respecto del nivel actual del terreno y se identificaron un total de seis capas estratigráficas naturales de distintos espesores y diversas características sedimentarias. El sedimento de la capa A es arenoso de origen eólico mientras que el de la capa $\mathrm{B}$ es arcilloso y compacto, de color rojizo. La capa $\mathrm{C}$ presenta sedimento areno-arcilloso con lentes de arena y pómez y la capa $\mathrm{D}$ es arcillosa compacta con presencia de carbones que forman lentes continuas y/o discontinuas y lentes centimétricas discontinuas de arena. Finalmente, la capa $\mathrm{E}$ presenta sedimento arenoso con carbones dispersos y la capa $\mathrm{F}$ es arcillosa compacta con carbones discontinuos y lentes centimétricas discontinuas de arena y pómez que rellenan oquedades. Se realizaron tres fechados radiocarbónicos, los cuales provienen de tres capas diferentes. En la capa F se obtuvo un fechado de $2210 \pm 80$ años AP (LP-3184, carbón); la capa D fue fechada en $1940 \pm 60$ años AP (LP-3182, carbón) y la capa C en $1790 \pm 70$ años AP (LP-3187, carbón). Con respecto a las calibraciones, para el fechado de la capa $\mathrm{F}$ se obtuvo el rango 358-136 cal a.C.; para el de la capa $\mathrm{D}$ el rango obtenido fue 44-203 cal d.C.; y para el de la capa $C$ se obtuvo el rango 214-376 cal d.C. (calibraciones a 1 sigma con el programa OxCal v4.2; Bronk Ramsey, 2009) (Figura 2). Así, se destaca que existe una concordancia estratigráfica entre los tres fechados en función de las profundidades de recuperación de las muestras. Esto corrobora la proposición de que 


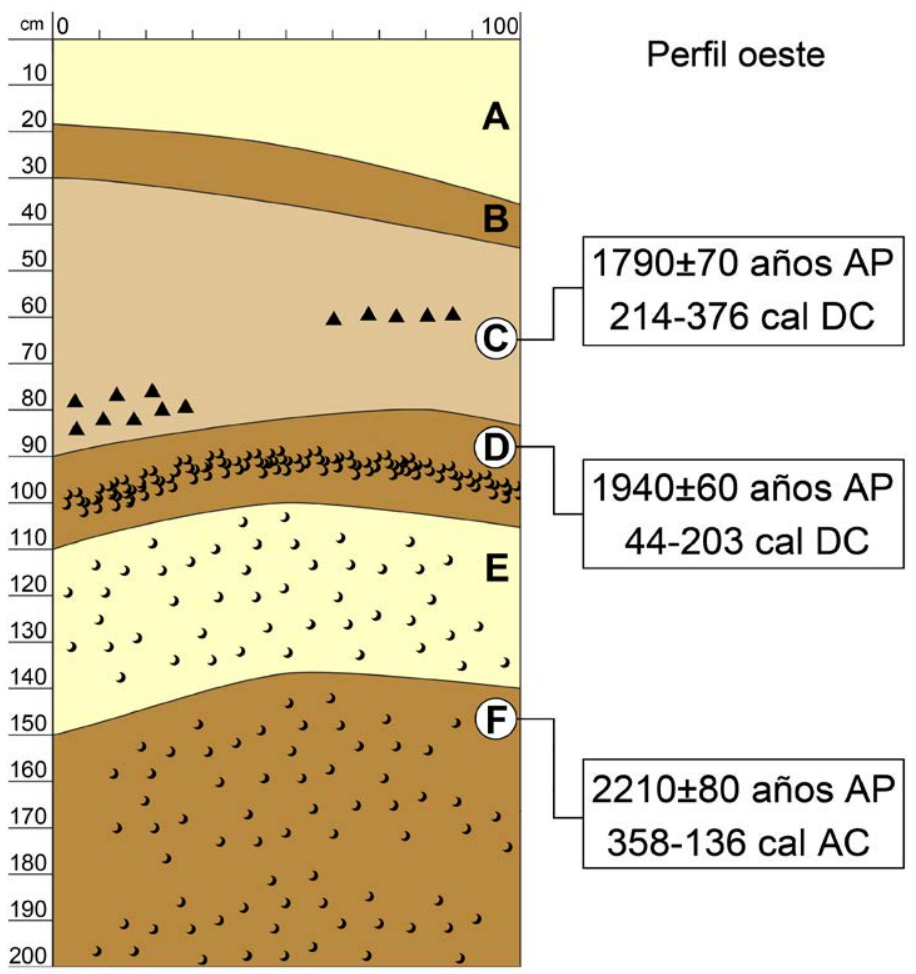

Figura 2. Perfil oeste de la excavación del basural de Palo Blanco.

el área no habría sido alterada por las excavaciones asistemáticas identificadas en zonas contiguas. Además, estos fechados representan los datos cronológicos más tempranos de ocupación humana documentada para la localidad arqueológica de Palo Blanco. En este sentido, por un lado, el fechado de la capa $\mathrm{F}$ es previo a cualquier ocupación de los $\mathrm{NH}$ identificados e intervenidos hasta el momento. Por otro lado, el fechado de la capa D es contemporáneo al NH7 (Ratto, comunicación personal, 2017) y el del C al NH1 (Miyano et al., 2015, tabla 1). Por lo tanto, el fechado de la capa F nos permite pensar acerca de la existencia de otros núcleos de la aldea aún no identificados y de mayor profundidad temporal que los registrados hasta la actualidad.

En la excavación del basural, y a lo largo de toda su secuencia, se recuperó una gran variedad de material arqueológico: 437 fragmentos cerámicos (principalmente tiestos aunque también "fichas" y un fragmento de figurina), 1.545 restos óseos de fauna, 98 fragmentos líticos (instrumentos y lascas), restos vegetales (marlos de maíz quemados y una semilla), tres restos malacológicos y un cincel de metal. Esta cantidad y diversidad de materiales y la información contextual anteriormente mencionada apoyan la idea de que el área intervenida se trata de la zona de basural identificada por Sempé (1976) al sur del NH4.

\section{Materiales y métodos}

Se analizaron 1.545 especímenes óseos provenientes de la excavación del basural. La principal característica de los basurales es que son depósitos de "grano grueso", es decir, que un mismo depósito presenta una acumulación de diversos eventos producidos por diferentes agentes (Mengoni Goñalons, 2006-2010). En este sentido, los basurales resultan informativos respecto a las estrategias humanas generales que le dieron origen. Con el objetivo de poder realizar comparaciones e identificar cambios y/o continuidades 
en las estrategias vinculadas al uso de los animales a través del tiempo, se conformaron tres conjuntos arqueofaunísticos que abarcan tres rangos temporales en función de los fechados radiocarbónicos del basural:

1. Conjunto arqueofaunístico de las capas E y F (Conjunto 1): está conformado por 567 especímenes óseos y fue asignado al bloque temporal 1 (BT1), comprendido entre los años 400 a.C. y 1 d.C.

2. Conjunto arqueofaunístico de las capas C y D (Conjunto 2): está compuesto por 694 especímenes óseos y fue asignado al bloque temporal 2 (BT2), comprendido entre los años 1 d.C. y 400 d.C.

3. Conjunto arqueofaunístico de las capas A y B (Conjunto 3): está conformado por 284 especímenes óseos. Las capas de las cuales provienen no poseen fechados radiocarbónicos de modo tal que este conjunto fue adscripto al bloque temporal 3 (BT3), el cual se adscribe cronológicamente a momentos posteriores a 400 d.C. Si bien su límite temporal no se puede precisar, la aldea de Palo Blanco en general no registra fechados posteriores al año 1000 d.C., momento en que habría ocurrido su abandono (Miyano et al., 2015; Ratto et al., 2015).

Los tres conjuntos arqueofaunísticos $(\mathrm{C} 1, \mathrm{C} 2$ y C3) fueron abordados de manera independiente pero considerando los mismos métodos. En primer lugar, la identificación anatómica y taxonómica de los especímenes óseos se realizó a partir de la comparación del material arqueofaunístico con las colecciones de referencia del Proyecto Arqueológico Chaschuil-Abaucán (Instituto de las Culturas, UBA-CONICET). Los especímenes identificados fueron asignados a distintas categorías taxonómicas considerando la presencia de zonas diagnósticas (Mengoni Goñalons, 1999) o distintos atributos morfológicos como tamaño, cualidades estructurales, curvatura y espesor (De Nigris, 2004). Todos los especímenes identificados anatómica y taxonómicamente conforman el Número de Especímenes Identificados por Taxón (NISP) (Lyman, 1994) cualquiera sea la resolución de la categoría taxonómica a la que fueran asignados (clase, orden, familia, género, etc.). Aquellos especímenes que no pudieron ser asignados a ninguna categoría taxonómica conforman el Número de Especímenes No Identificables (NID) (Mengoni Goñalons, 1999). La suma del NISP y el NID compone el Número Total de Especímenes (NSP) (Grayson, 1991).

En segundo lugar, se determinó la riqueza taxonómica (NTAXA) la cual se calcula a partir de la suma de las categorías taxonómicas que no se superponen (Grayson, 1984, 1991), considerando entre los mamíferos el nivel taxonómico de familia. Además, se calculó el Índice de Artiodáctilos (IA) (Broughton, 1994) a partir de la siguiente fórmula: NISP artiodáctilos / NISP artiodáctilos + NISP vertebrados pequeños. En este caso, la categoría "vertebrados pequeños" comprende a los especímenes de roedores, dasipódidos y aves. El IA es un índice de abundancia relativa que permite dar cuenta de la importancia de los artiodáctilos dentro de un conjunto: un valor cercano a 1 indica la predominancia de artiodáctilos en el conjunto mientras que un valor cercano a 0 indica que los artiodáctilos poseen poca representación.

En tercer lugar, el subconjunto Camelidae fue analizado mediante distintos métodos que buscaron identificar especies de camélidos, medir la abundancia anatómica, evaluar el aporte de distintas partes esqueletarias con cantidades variables de recursos primarios asociados y construir perfiles etarios.

El análisis cuantitativo de las medidas de elementos del esqueleto apendicular de camélidos permite diferenciar especies grandes (guanaco y llama) de pequeñas (vicuña y alpaca). La presencia de alpaca en el pasado prehispánico del noroeste argentino 
es cuestionada dado que: a) las condiciones ambientales de relativa sequedad de la puna argentina no son aptas para su cría (Mengoni Goñalons, 2008) y b) no existe evidencia zooarqueológica concluyente respecto a la presencia de este camélido en dicha región (Mengoni Goñalons y Yacobaccio, 2006). De este modo, durante momentos precolombinos solo habrían habitado vicuñas, guanacos y llamas. Los análisis osteométricos se realizaron a partir del empleo de la técnica de diferencia de logaritmos $(d l)$ propuesta por Meadow (1987). A diferencia de los análisis osteométricos multivariados, esta técnica permite comparar medidas provenientes de distintos elementos de manera simultánea y, dado que solo se utiliza una medida por hueso, se maximiza la cantidad de especímenes óseos potencialmente informativos respecto a su adscripción taxonómica a nivel género y/o especie (Grant, 2010). Así, se seleccionó una dimensión por cada elemento ${ }^{1}$ y se la comparó con su equivalente tomada de un camélido de referencia actual (guanaco de las Cumbres Calchaquíes analizado por Elkin y Mengoni Goñalons; Mengoni Goñalons, comunicación personal, 2016). La comparación se realizó mediante la fórmula $d l=\log (x)-\log (z)=\log (x / z)$; donde $x$ es la medida del espécimen arqueológico y $z$ su equivalente del guanaco actual. Siguiendo a López (2003), se consideró que un valor de $d l$ menor a $-0,02$ sugiere la presencia de vicuña (Vicugna vicugna); un valor mayor a 0,02 indica la presencia de un camélido de tamaño grande asignable a llama (Lama glama); y un valor entre - 0,02 y 0,02 supone que el espécimen podría pertenecer tanto a llama como a guanaco (Lama sp.), utilizándose la categoría "llama-guanaco" para englobar estos casos de tamaño intermedio.

La abundancia anatómica de Camelidae se calculó a partir de distintas medidas. En primera instancia, se calculó el Número Mínimo de Elementos (MNE) (Binford, 1984) a partir de la combinación de los métodos de zonas diagnósticas (Mengoni Goñalons, 1999) y la suma de fracciones (Klein y Cruz-Uribe, 1984) considerando variables tales como lateralidad, estado de fusión y tamaño relativo de los especímenes así como también criterios osteométricos con el objetivo de obtener la mayor discriminación posible. Posteriormente, se calculó el Número Mínimo de Unidades Anatómicas (MAU) que luego fue estandarizado en una escala de 0 a 100 (\%MAU) (Binford, 1978, 1984). El MNE se empleó también para calcular otras medidas. Por un lado, el MNE se utilizó para calcular las Partes Esqueletarias Básicas (PEB) (Yacobaccio, Madero, Malmierca y Reigadas, 1997-1998). Las PEB se calculan a partir de la suma de las proporciones de cada MNE agrupados en diez zonas del esqueleto: cabeza (cráneo y mandíbula), columna (vértebras cervicales, torácicas, lumbares y sacro), costillar (costillas y esternebras), pelvis, escápula, pata delantera (húmero y radioulna), pata trasera (fémur y tibia), pie delantero (carpianos y metacarpo), pie trasero (tarsianos y metatarso) y falanges. Esta medida permite observar la representación de las distintas zonas esqueletarias, las cuales se corresponden con las unidades en que los pastores actuales trozan sus animales. Por otro lado, a partir del MNE se calculó la relación entre el MNE observado en cada conjunto (MNEO) y el MNE esperado para siete regiones anatómicas de un esqueleto completo de camélido (MNEE). El criterio para definir estas siete regiones fue la cantidad relativa de carne y médula que ofrecen (De Nigris 2004), de modo tal que esta medida permite realizar una aproximación a la utilidad nutricional de los conjuntos arqueofaunísticos. El esqueleto se dividió en: a) columna (vértebras y sacro; MNEE $=27$ ), costillar (costillas y esternebras; MNEE = 30) y cinturas (escápula e innominado; $\mathrm{MNEE}=4$ ), las cuales ofrecen solo carne; $b$ ) extremidades superiores (húmero y fémur: $\mathrm{MNEE}=4$ ), las cuales brindan abundante carne y médula; $c$ ) extremidades medias (radioulna y tibia; $\mathrm{MNEE}=4$ ), las que otorgan poca carne y médula; $\mathrm{d}$ ) extremidades inferiores (metapodios y falanges 1 y $2 ; \mathrm{MNEE}=$ 20), las que ofrecen solo médula en proporciones moderadas-bajas; y e) cabeza (cráneo y hemimandíbula; $\mathrm{MNEE}=3$ ), la cual contiene órganos ricos en grasa. La relación entre MNEE y MNEO se estandarizó en una escala de 0 a 100. Complementariamente a lo último, se correlacionó el \%MAU con dos índices de utilidad económica que realizan un ranking de los elementos óseos en función de la cantidad de recursos que ofrecen:
2. Las dimensiones seleccionadas fueron: ancho de la cavidad glenoidea de la escápula (LG, von den Driesch, 1976; SCA165, Kent, 1982), ancho máximo del extremo distal de la tibia (Bd von den Driesch, 1976; TIB102, Kent, 1982), largo máximo del calcáneo (GL, von den Driesch, 1976), ancho máximo de la superficie articular proximal del metacarpo (MCARP59, Kent, 1982) y del metatarso (MTAR3o, Kent, 1982), ancho máximo del extremo distal del metapodio (Bd, von den Driesch, 1976), espesor del extremo proximal de la falange $1\left(\mathrm{FP}_{1} \mathrm{~V}_{3}\right.$, Kent, 1982) y la falange 2 ( $\mathrm{P}_{2} \mathrm{~V} 8$, Kent, 1982). 
Índice de Carne (Mengoni Goñalons, 1991) e Índice de Médula (Mengoni Goñalons, 1996). Además, se correlacionó el \%MAU con el Îndice de Secado (De Nigris y Mengoni Goñalons, 2005), el cual ordena a los distintos elementos óseos en función de su aptitud intrínseca para el proceso de secado. Mientras que los primeros dos índices se emplean para dar cuenta de contextos de consumo inmediato, el último refiere a circunstancias de consumo diferido. Con el objetivo de controlar que los perfiles anatómicos no sean el resultado de una supervivencia diferencial dependiente de la densidad de los huesos, se correlacionó el \%MAU de Camelidae con los valores de densidad mineral ósea propuestos por Elkin (1995). Las correlaciones entre el \%MAU y los cuatro índices mencionados se realizaron mediante rho de Spearman.

La construcción de perfiles etarios de Camelidae se realizó a partir de dos métodos complementarios. Para ello, en primer lugar, se calculó el Î́ndice de Camélidos Subadultos (ISA) a partir de la razón entre el número de especímenes sin epifizar (NISP NF) sobre el total de especímenes no fusionados (NISP NF) y fusionados (NISP F) del subconjunto Camelidae. Un valor cercano a 1 indica la predominancia de camélidos subadultos mientras que un valor cercano a 0 indica una predominancia de camélidos adultos (Belotti López de Medina, 2015). En segundo lugar, y para refinar esta primera aproximación a la estructura de edad de los conjuntos, se construyeron perfiles etarios a partir de aquellos especímenes óseos fusionados o no fusionados que por su tamaño podrían pertenecer a llama y se excluyeron los de tamaño pequeño que con seguridad pertenecerían a vicuña con el objetivo de dar cuenta de estrategias en el manejo de camélidos domésticos. Específicamente, se consideró el estado de fusión de ciertos centros de osificación presentes en la escápula, el innominado, los huesos largos y las primeras falanges los cuales se agrupan en tres etapas no superpuestas: fusión temprana (12 a 18 meses), fusión intermedia (18 a 36 meses) y fusión tardía ( 36 a 48 meses) (Mengoni Goñalons, 2013, tabla 3). Así, la construcción de los perfiles etarios se realizó a partir de las frecuencias de NISP -F o NF- para cada una de las tres etapas anteriormente mencionadas. El sesgo que puede existir en la construcción de estos perfiles se debe a la posible inclusión de especímenes correspondientes a guanaco (Mengoni Goñalons, 2013).

Finalmente, se registraron los distintos tipos de modificaciones presentes en el NISP. Por un lado, se analizaron las marcas de origen antrópico relacionadas al procesamiento de los animales tales como huellas de corte, raspado, machacado y percusión (Lyman, 1994, 2008; Mengoni Goñalons, 1999) así como también aquellas marcas que sugieran la formatización de restos óseos: lascado, pulido de superficies, raspado y estrías producto del uso, extremos punzantes y marcado perimetral (Izeta 2007; Izeta et al., 2013). Además, se registró el color de los especímenes óseos para dar cuenta de la acción térmica (Mengoni Goñalons, 1999). Por otro lado, se analizaron las marcas causadas por otro tipo de agentes no antrópicos: carnívoros y roedores (Mengoni Goñalons, 1999). Por último, se registraron los estadios de meteorización (Behrensmeyer, 1978).

\section{Resultados}

\section{Abundancia y diversidad taxonómica}

3. Las cuatro familias de mamíferos representadas tanto en el $\mathrm{C} 1$ como en el C2 son Camelidae, Dasypodidae, Chinchillidae y Caviidae. Si se consideran los especímenes asignados a la clase Aves, el NTAXA asciende a 5 dado que resulta imposible que dichos restos se traslapen con las categorías de la clase Mammalia.

Los restos óseos de camélidos son los más frecuentes en los tres conjuntos del basural. La predominancia de Camelidae es mayor aún si se le suman los especímenes de Artiodactyla, dado que no se registró otro taxón de este orden (como Cervidae) (Tabla 1). Sin embargo, hay diferencias en cuanto a la riqueza taxonómica y la abundancia relativa de los artiodáctilos en los tres conjuntos. En este sentido, para los momentos más tempranos (C1), se registra un NTAXA de $5^{2}$ y un IA de 0,67. Posteriormente, en el C2, se mantienen la misma riqueza taxonómica aunque el IA aumenta a 0,78 dado 


\begin{tabular}{|l|c|c|c|c|c|c|}
\hline \multirow{2}{*}{ Taxón } & \multicolumn{2}{|c|}{ C1 $_{1}$} & \multicolumn{2}{c|}{ C2 $_{2}$} & \multicolumn{2}{c|}{$\mathbf{C}_{\mathbf{3}}$} \\
\cline { 2 - 7 } & N & \% & N & \% & N & \% \\
\hline MAMMALIA (indeterminado) & 25 & 7,40 & 10 & 2,65 & 1 & 0,73 \\
Artiodactyla & 65 & 19,23 & 85 & 22,55 & 57 & 41,61 \\
Camelidae & 144 & 42,60 & 200 & 53,05 & 79 & 57,66 \\
Dasypodidae & 70 & 20,71 & 56 & 14,85 & - & - \\
Rodentia & 25 & 7,40 & 8 & 2,12 & - & - \\
Chinchillidae & 2 & 0,59 & 6 & 1,59 & - & - \\
Caviidae & 3 & 0,89 & 3 & 0,80 & - & - \\
AVES (indeterminado) & 4 & 1,18 & 9 & 2,39 & - & - \\
\hline Total NISP & 338 & $\mathbf{1 0 0 , 0 0}$ & 377 & $\mathbf{1 0 0 , 0 0}$ & $\mathbf{1 3 7}$ & $\mathbf{1 0 0 , 0 0}$ \\
Total NID & $\mathbf{2 2 9}$ & - & 317 & - & $\mathbf{1 4 7}$ & - \\
NSP & 567 & - & $\mathbf{6 9 4}$ & - & $\mathbf{2 8 4}$ & - \\
\hline
\end{tabular}

Tabla 1. Diversidad taxonómica del basural de Palo Blanco.

que la frecuencia de los vertebrados pequeños disminuye. Finalmente, en el C3 solo se registraron artiodáctilos/camélidos de modo tal que el IA y el NTAXA poseen valores de 1. La baja diversidad taxonómica del C3 puede estar relacionada con la cantidad de especímenes óseos, la cual es menor a la del C1 y C2. Sin embargo, y más allá de esto último, se observa un incremento de la importancia de los artiodáctilos a través del tiempo en detrimento de los vertebrados pequeños (roedores, dasipódidos y aves).

Respecto a la identificación interespecífica de camélidos, se observa que para el C1 solo se registran especímenes asignables a llama $(n=5)$, algunos de tamaño muy grande como aquellos del intervalo 0,08-0,09. En el C2 se registraron especímenes asignables a llama $(n=2)$ y a llama-guanaco $(n=1)$. Finalmente, en el C3 se observa la mayor diversidad de tamaños de camélidos presentes en el basural, registrándose especímenes asignables a llama $(\mathrm{n}=5)$ (uno considerablemente grande en el intervalo 0,11-0,12), a llama-guanaco $(\mathrm{n}=1)$ y a vicuña $(\mathrm{n}=2)$, camélido silvestre alóctono (Figura 3$)$. $\mathrm{Si}$ bien la muestra total es pequeña $(n=16)$, es posible identificar un incremento en la diversidad de tamaño de camélidos a través del tiempo y la introducción de vicuñas en momentos posteriores al 400 d.C.

\section{Representación de partes esqueletarias de camélidos}

La gran mayoría de las partes esqueletarias de camélidos se encuentran representadas en los dos conjuntos más tempranos $(\mathrm{C} 1$ y C2) mientras que en el C3 se destaca la ausencia de varios elementos óseos (principalmente, vértebras, carpianos y tarsianos). Si se considera el NISP, se observa una predominancia de especímenes del esqueleto axial por sobre el apendicular en los tres conjuntos: $61,81 \%$ en el C1, 72,50\% en el C2 y 60,76\% en el C3. En cambio, si se considera el MNE, la representación del esqueleto axial es relativamente menor en los tres bloques temporales ( $43,28 \%$ en el C1, 55,07\% en el C2 y $45,95 \%$ en el C3) lo cual posiblemente se relacione con una menor fragmentación del esqueleto apendicular. Finalmente, mientras que el cráneo posee el MAU más alto en el C1, en los Conjuntos 2 y 3 el valor de MAU más elevado corresponde a la tibia (Tabla 2).

La representación de las PEB muestra ciertas tendencias compartidas por los tres bloques temporales y algunas diferencias. Las similitudes se observan en respecto de las frecuencias de las partes del esqueleto axial. En este sentido, y al realizar la comparación con la curva esperada para el esqueleto completo de un camélido, se observa una mayor representación del costillar y una subrrepresentación de la columna. Las diferencias se 


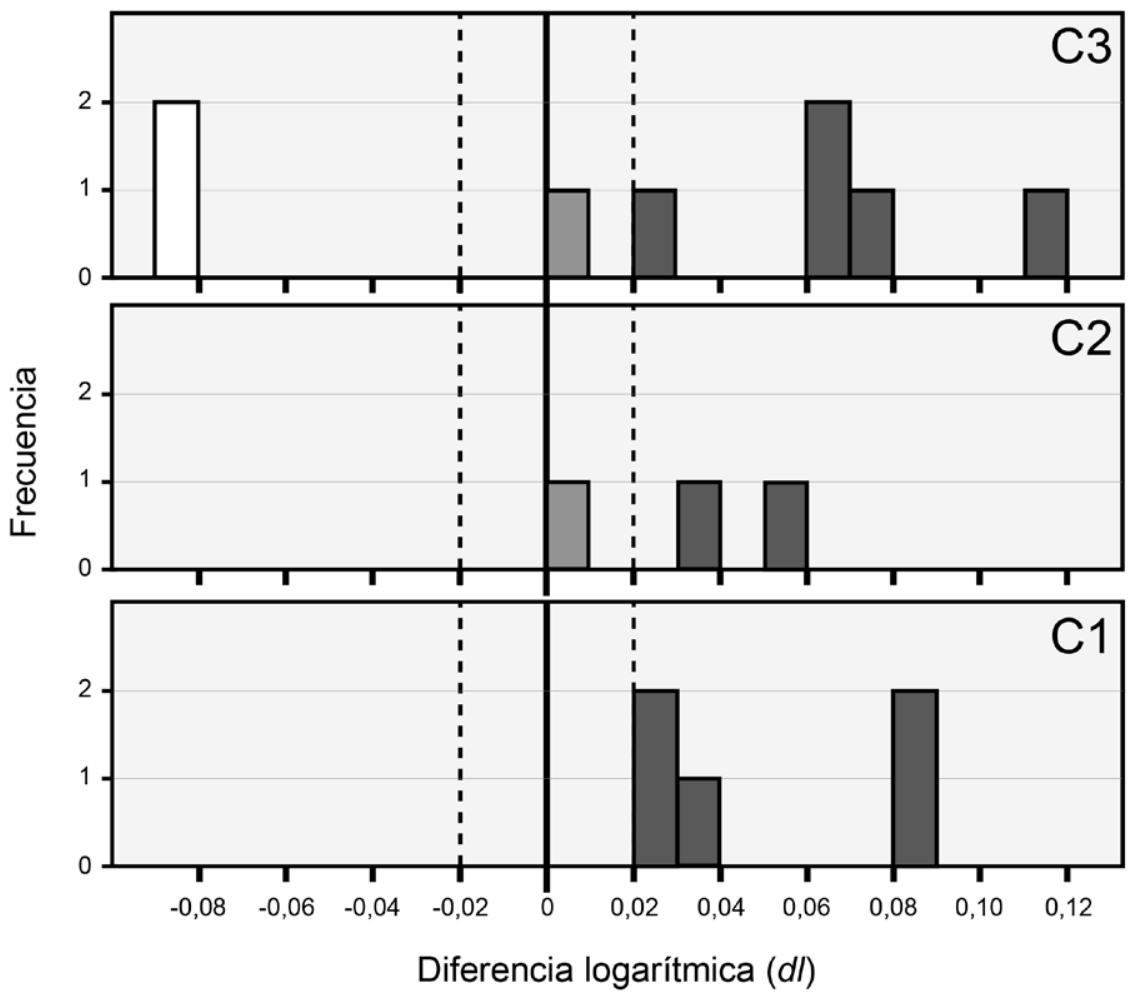

Figura 3. Diferencia de logaritmos entre los casos arqueológicos del basural de Palo Blanco y el camélido de referencia actual.

observan respecto a las frecuencias de las partes del esqueleto apendicular. Mientras que la curva del C1 muestra frecuencias similares a la esperada para el esqueleto completo de un camélido, las curvas de los Conjuntos 2 y 3 exhiben ciertas oscilaciones respecto a ella. Por un lado, en el C2 se observa una mayor frecuencia de la pata trasera y una subrrepresentación de los huesos del autopodio (pies y falanges). Por otro lado, en el C3 se destaca una mayor frecuencia de ambas patas (delantera y trasera) en detrimento de ambos pies y, a diferencia del C2, las falanges poseen mayor representación (Figura 4A).

\section{Utilidad económica de las partes esqueletarias de camélidos y consumo}

La relación $\mathrm{MNEO} / \mathrm{MNEE}$ de las siete regiones anatómicas de camélidos presenta similitudes y diferencias entre los tres conjuntos. Respecto a las similitudes, se destaca que el cráneo y la columna poseen una representación relativamente similar, muy alta en el caso del cráneo y muy baja en el caso de la columna. Las principales diferencias se observan en las frecuencias del costillar, las cinturas y las extremidades superiores, medias e inferiores. En este sentido, en el $\mathrm{C} 1$ ninguna de estas cinco regiones registra una frecuencia menor al 60\%, siendo las cinturas y las extremidades superiores las más abundantes. En el C2, las extremidades superiores y medias son las más abundantes, seguidas por el costillar. Finalmente, el C3 posee una curva similar a la del C2, aunque la única diferencia se observa en la frecuencia del costillar la cual disminuye al $30 \%$ (Figura 4B).

Para complementar los datos presentados anteriormente, se correlacionó, en primer lugar, el \%MAU con los valores de densidad mineral ósea propuestos por Elkin (1995). En este sentido, la correlación entre el \%MAU del esqueleto axial del C3 y los valores de densidad mineral ósea fue alta, positiva y significativa. Esto sugiere que ciertos procesos de formación podrían estar actuando en la preservación diferencial de los huesos de 


\begin{tabular}{|c|c|c|c|c|c|c|c|c|c|c|c|c|}
\hline \multirow{2}{*}{ Parte esqueletaria } & \multicolumn{4}{|c|}{$\mathrm{C}_{1}$} & \multicolumn{4}{|c|}{$\mathrm{C}_{2}$} & \multicolumn{4}{|c|}{$\mathrm{C}_{3}$} \\
\hline & NISP & MNE & MAU & \%MAU & NISP & MNE & MAU & \%MAU & NISP & MNE & MAU & \%MAU \\
\hline Dientes sueltos & 4 & - & - & - & 16 & - & - & - & 1 & - & - & - \\
\hline Cráneo & 20 & 2 & 2,00 & 100,00 & 28 & 1 & 1,00 & 50,00 & 3 & 1 & 1,00 & 66,67 \\
\hline Mandíbula & 1 & 1 & 0,50 & 25,00 & 2 & 2 & 1,00 & 50,00 & 3 & 2 & 1,00 & 66,67 \\
\hline Hioides & 4 & 1 & 0,50 & 25,00 & 2 & 1 & 0,50 & 25,00 & - & - & 0,00 & 0,00 \\
\hline Atlas & - & - & 0,00 & 0,00 & - & - & 0,00 & 0,00 & 1 & 1 & 1,00 & 66,67 \\
\hline Axis & - & - & 0,00 & 0,00 & - & - & 0,00 & 0,00 & - & - & 0,00 & 0,00 \\
\hline Cervicales & 7 & 3 & 0,60 & 30,00 & 6 & 1 & 0,20 & 10,00 & 1 & 1 & 0,20 & 13,33 \\
\hline Torácicas & 4 & 2 & 0,17 & 8,33 & 2 & 1 & 0,08 & 4,17 & - & - & 0,00 & 0,00 \\
\hline Lumbares & 2 & 1 & 0,14 & 7,14 & 6 & 3 & 0,43 & 21,43 & - & - & 0,00 & 0,00 \\
\hline Sacro & - & - & 0,00 & 0,00 & - & - & 0,00 & 0,00 & - & - & 0,00 & 0,00 \\
\hline Cartílago intercostal & - & - & - & - & 3 & - & - & - & - & - & - & - \\
\hline Innominado & 3 & 1 & 0,50 & 25,00 & 1 & 1 & 0,50 & 25,00 & 3 & 1 & 0,50 & 33,33 \\
\hline Costillas & 44 & 18 & 0,75 & 37,50 & 78 & 27 & 1,13 & 56,25 & 36 & 11 & 0,46 & 30,56 \\
\hline Esternebras & - & - & 0,00 & 0,00 & 1 & 1 & 0,17 & 8,33 & - & - & 0,00 & 0,00 \\
\hline Escápula & 4 & 3 & 1,50 & 75,00 & 2 & 1 & 0,50 & 25,00 & 3 & 1 & 0,50 & 33,33 \\
\hline Húmero & 5 & 2 & 1,00 & 50,00 & 4 & 1 & 0,50 & 25,00 & 4 & 2 & 1,00 & 66,67 \\
\hline Radioulna & 3 & 2 & 1,00 & 50,00 & 5 & 2 & 1,00 & 50,00 & 2 & 2 & 1,00 & 66,67 \\
\hline Carpianos & 6 & 6 & 0,43 & 21,43 & 2 & 2 & 0,14 & 7,14 & - & - & 0,00 & 0,00 \\
\hline Fémur & 7 & 2 & 1,00 & 50,00 & 7 & 4 & 2,00 & 100,00 & 3 & 2 & 1,00 & 66,67 \\
\hline Rótula & - & - & 0,00 & 0,00 & 3 & 3 & 1,50 & 75,00 & - & - & 0,00 & 0,00 \\
\hline Tibia & 1 & 1 & 0,50 & 25,00 & 11 & 4 & 2,00 & 100,00 & 9 & 3 & 1,50 & 100,00 \\
\hline Tarsianos & 3 & 3 & 0,30 & 15,00 & 2 & 2 & 0,20 & 10,00 & - & - & 0,00 & 0,00 \\
\hline Astrágalo & - & - & 0,00 & 0,00 & - & - & 0,00 & 0,00 & 1 & 1 & 0,50 & 33,33 \\
\hline Calcáneo & 2 & 2 & 1,00 & 50,00 & 1 & 1 & 0,50 & 25,00 & - & - & 0,00 & 0,00 \\
\hline Metacarpo px & - & - & 0,00 & 0,00 & 3 & 3 & 1,50 & 75,00 & 1 & 1 & 0,50 & 33,33 \\
\hline Metatarso px & 1 & 1 & 0,50 & 25,00 & - & - & 0,00 & 0,00 & - & - & 0,00 & 0,00 \\
\hline Metapodio ind & 7 & 2 & 0,50 & 25,00 & 9 & 2 & 0,50 & 25,00 & 1 & 1 & 0,25 & 16,67 \\
\hline Falange 1 & 3 & 3 & 0,38 & 18,75 & 3 & 3 & 0,38 & 18,75 & 4 & 4 & 0,50 & 33,33 \\
\hline Falange 2 & 9 & 7 & 0,88 & 43,75 & 2 & 2 & 0,25 & 12,50 & 3 & 3 & 0,38 & 25,00 \\
\hline Falange 3 & 2 & 2 & 0,25 & 12,50 & - & - & 0,00 & 0,00 & - & - & 0,00 & 0,00 \\
\hline Sesamoideos & 2 & 2 & - & - & 1 & 1 & - & - & - & - & - & - \\
\hline Total Axial & 89 & 29 & & & 145 & 38 & & & 48 & 17 & & \\
\hline Total Apendicular & 55 & 38 & & & 55 & 31 & & & 31 & 20 & & \\
\hline Total & 144 & 67 & & & 200 & 69 & & & 79 & 37 & & \\
\hline
\end{tabular}

Tabla 2. NISP, MNE, MAU y \%MAU de cada parte esqueletaria de Camelidae de los conjuntos arqueofaunísticos del basural de Palo Blanco.

este subconjunto. El resto de las correlaciones son bajas y no significativas de modo que no habría supervivencia diferencial dependiente de la densidad ósea en los demás conjuntos (Tabla 3). En segundo lugar, se correlacionó el \%MAU con los índices de Carne (Mengoni Goñalons, 1991), de Médula (Mengoni Goñalons, 1996) y de Secado (De Nigris y Mengoni Goñalons, 2005). Por un lado, las correlaciones realizadas con el Índice de Carne, tanto para el esqueleto completo como para el esqueleto axial, fueron bajas-moderadas, positivas (con excepción de la del esqueleto axial del C3 que fue negativa) y no significativas. Respecto a las correlaciones realizadas con el esqueleto apendicular, en los tres casos fueron moderadas-altas, positivas y significativas. Esto 


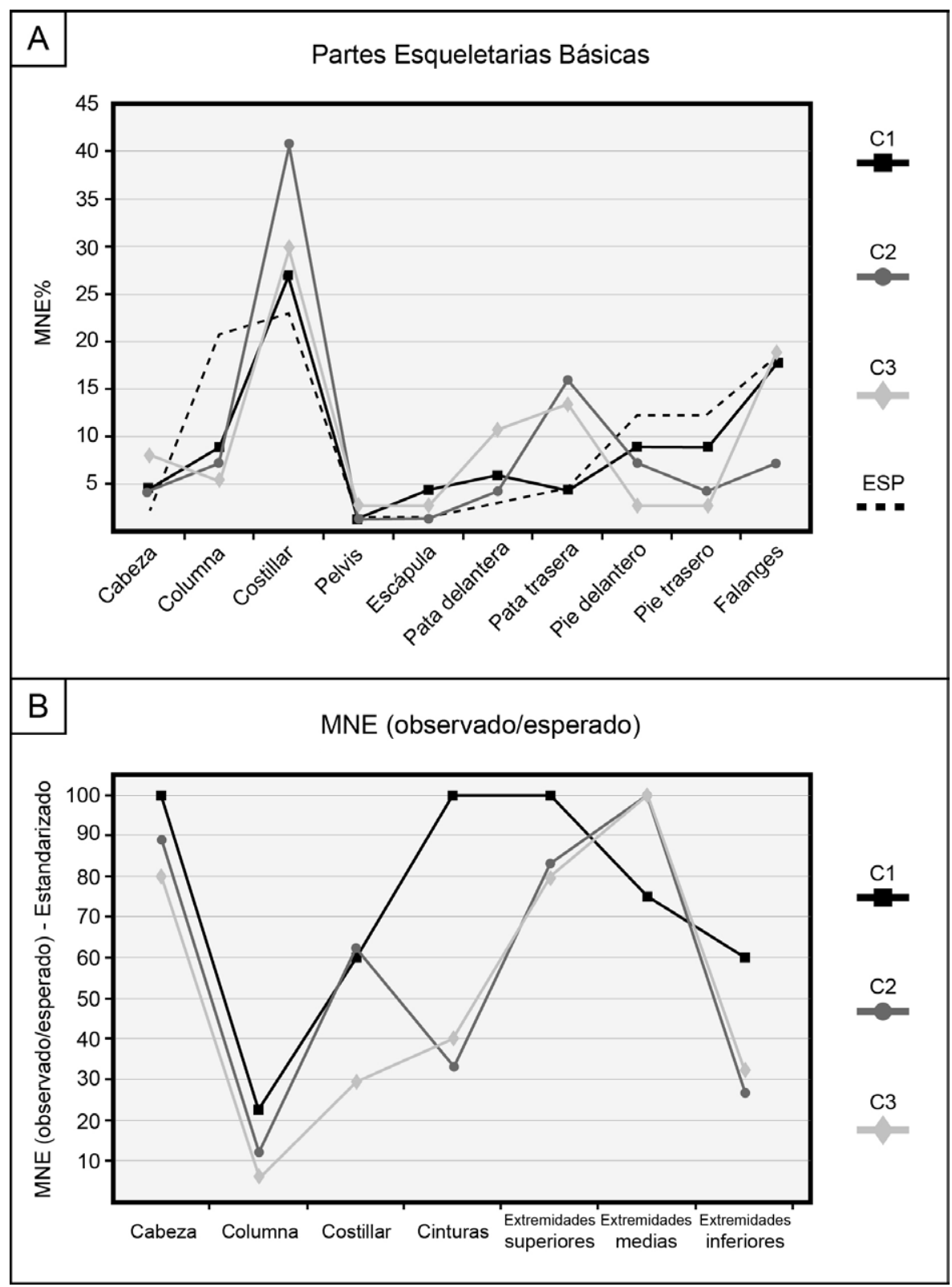

Figura 4. Abundancia anatómica de Camelidae. A) Representación de las partes esqueletarias básicas de Camelidae. B) Relación MNEO/MNEE de siete regiones anatómicas de Camelidae.

sugiere que en los tres casos la frecuencia de huesos del esqueleto apendicular puede explicarse por el consumo inmediato de carne. Por otro lado, las correlaciones realizadas con el Îndice de Médula fueron en los tres casos moderadas-altas y positivas, aunque solo en el caso del C2 fue significativa. De este modo, en el caso del C2 el conjunto de huesos del esqueleto apendicular no solo puede explicarse por el consumo inmediato de carne sino también por el de médula. Finalmente, se correlacionó el \%MAU con el Índice de Secado. En los tres casos las correlaciones fueron moderadas-altas y negativas y en los casos de los Conjuntos 1 y 3 fueron significativas. Esto sugiere que las unidades anatómicas que resultan más apropiadas para la producción de charki y su posterior consumo diferido, se encuentran escasamente representadas en estos dos contextos. Sin embargo, y como se señaló anteriormente, el esqueleto axial del C3 podría haber sufrido procesos destructivos postdepositacionales de modo que resulta problemático 


\begin{tabular}{|c|c|c|c|c|c|c|c|c|}
\hline \multirow{2}{*}{ Conjunto } & \multicolumn{3}{|c|}{ Índice de Carne } & \multirow{2}{*}{$\begin{array}{c}\begin{array}{c}\text { Índice de } \\
\text { Médula }\end{array} \\
\begin{array}{c}\text { Huesos largos } \\
\text { falanges } 1\end{array}\end{array}$} & \multirow{2}{*}{$\begin{array}{c}\begin{array}{c}\text { Índice de } \\
\text { Secado }\end{array} \\
\text { Esqueleto } \\
\text { Completo }\end{array}$} & \multicolumn{3}{|c|}{ Densidad mineral ósea } \\
\hline & $\begin{array}{l}\text { Esqueleto } \\
\text { Completo }\end{array}$ & $\begin{array}{c}\text { Esqueleto } \\
\text { Axial }\end{array}$ & $\begin{array}{l}\text { Esqueleto } \\
\text { Apendicular }\end{array}$ & & & $\begin{array}{l}\text { Esqueleto } \\
\text { Completo }\end{array}$ & $\begin{array}{c}\text { Esqueleto } \\
\text { Axial }\end{array}$ & $\begin{array}{l}\text { Esqueleto } \\
\text { Apendicular }\end{array}$ \\
\hline $\mathrm{C}_{1}$ & $\begin{array}{l}r s=0,16 \\
p>0,05\end{array}$ & $\begin{array}{l}r s=0,26 \\
p>0,05\end{array}$ & $\begin{array}{l}r s=0,55 \\
p<0,05\end{array}$ & $\begin{array}{l}r s=0,62 \\
p>0,05\end{array}$ & $\begin{aligned} r s & =-0,57 \\
p & <0,05\end{aligned}$ & $\begin{array}{l}r s=0,3 \\
p>0,05\end{array}$ & $\begin{array}{l}r s=0,24 \\
p>0,05\end{array}$ & $\begin{array}{l}r s=0,31 \\
p>0,05\end{array}$ \\
\hline $\mathrm{C}_{2}$ & $\begin{array}{l}r s=0,4 \\
p>0,05\end{array}$ & $\begin{array}{l}r s=0,41 \\
p>0,05\end{array}$ & $\begin{array}{l}r s=0,71 \\
p<0,01\end{array}$ & $\begin{array}{l}r s=0,88 \\
p<0,05\end{array}$ & $\begin{aligned} r s & =-0,49 \\
p & >0,05\end{aligned}$ & $\begin{array}{l}r s=0,28 \\
p>0,05\end{array}$ & $\begin{array}{l}r s=-0,1 \\
p>0,05\end{array}$ & $\begin{array}{l}r s=0,24 \\
p>0,05\end{array}$ \\
\hline $\mathrm{C}_{3}$ & $\begin{array}{l}r s=0,16 \\
p>0,05\end{array}$ & $\begin{aligned} r s & =-0,48 \\
p & >0,05\end{aligned}$ & $\begin{array}{l}r s=0,7 \\
p<0,01\end{array}$ & $\begin{array}{l}r s=0,76 \\
p>0,05\end{array}$ & $\begin{aligned} r s & =-0,79 \\
p & <0,01\end{aligned}$ & $\begin{array}{l}r s=0,19 \\
p>0,05\end{array}$ & $\begin{array}{l}r s=0,71 \\
p<0,05\end{array}$ & $\begin{array}{l}r s=-0,13 \\
p>0,05\end{array}$ \\
\hline
\end{tabular}

Tabla 3. Correlaciones entre las frecuencias anatómicas de camélido (\%MAU) y los índices de utilidad y densidad mineral ósea.

determinar si la conformación de dicho conjunto responde a una estrategia humana de selección de partes o a factores densitodependietes.

\section{Perfiles etarios de camélidos}

Los tres conjuntos exhiben ciertas diferencias en la estructura etaria de Camelidae. Por un lado, el ISA del C1 es de 0,60 (NISP NF = 12; NISP F $=8$ ), marcando una cierta predominancia de camélidos subadultos. Si consideramos las etapas de fusión, se observa un perfil complejo dado que para la categoría etaria de fusión temprana solo se registran especímenes óseos no fusionados, indicando la muerte de animales menores de 12-18 meses de edad. Sin embargo, en la segunda categoría etaria sí se registran especímenes óseos fusionados y, además, éstos son más abundantes que los no fusionados para la misma categoría. Finalmente, la última categoría etaria solo exhibe especímenes no fusionados (Tabla 4). Este perfil etario podría explicarse mediante la matanza selectiva de animales jóvenes, posiblemente de animales en edad óptima como productores de carne (2-3 años).

Por otro lado, el ISA del C2 es el más cercano a 1 de los tres conjuntos con un valor de 0,74 (NISP NF $=14$; NISP F $=5$ ), marcando una predominancia de camélidos subadultos. El perfil etario resulta más claro que el anterior y se observa que solo un 25\% de los animales habrían sobrevivido a los 18-36 meses de edad. Así, entre un $75-100 \%$ de los camélidos murieron antes de los 48 meses de edad (Tabla 4). De este modo, y al igual que lo sucedido en el C1, el sacrificio de los camélidos se habría centrado principalmente en individuos jóvenes.

Finalmente, el C3 posee el ISA más cercano a 0 de los tres conjuntos con un valor de 0,32 (NISP NF = 6; NISP F = 13) lo cual indicaría un predominio de camélidos adultos. Además, el perfil etario exhibe una tasa de supervivencia mayor si se los compara con los otros dos perfiles (Tabla 4). En este sentido, entre un 50-67\% de los animales vivieron más allá de los 48 meses de edad lo cual puede relacionarse con la conservación de animales vivos para el aprovechamiento de su fibra y/o su capacidad de carga así como también con el mantenimiento de parte del rebaño como reserva de carne.

\section{Modificaciones óseas}

Se analizaron marcas de origen antrópico en todos los especímenes óseos, exceptuando fragmentos de cartílago intercostal y dientes. En este sentido, en los tres conjuntos se registraron marcas sobre especímenes de Artiodactyla y Camelidae que sugieren el procesamiento humano. Respecto al subconjunto Camelidae, en el C1 se registró la frecuencia más alta de marcas de origen antrópico sobre el total del NISP para este 


\begin{tabular}{|c|c|c|c|c|c|c|c|c|c|c|c|c|}
\hline \multirow[b]{2}{*}{ Etapas } & \multicolumn{4}{|c|}{$\mathrm{C}_{1}$} & \multicolumn{4}{|c|}{$\mathrm{C}_{2}$} & \multicolumn{4}{|c|}{$C_{3}$} \\
\hline & $\begin{array}{l}\text { NISP } \\
F\end{array}$ & $\begin{array}{l}\text { NISP } \\
\text { NF }\end{array}$ & $\begin{array}{c}\% \\
\text { SUP }\end{array}$ & $\%$ MOR & $\begin{array}{l}\text { NISP } \\
F\end{array}$ & $\begin{array}{l}\text { NISP } \\
\text { NF }\end{array}$ & $\begin{array}{l}\% \\
\text { SUP }\end{array}$ & $\%$ MOR & $\begin{array}{c}\text { NISP } \\
F\end{array}$ & $\begin{array}{l}\text { NISP } \\
\text { NF }\end{array}$ & $\begin{array}{l}\% \\
\text { SUP }\end{array}$ & $\%$ MOR \\
\hline $\begin{array}{l}\text { Fusión temprana } \\
(<12-18 \text { meses })\end{array}$ & o & 3 & 0,00 & 100,00 & - & - & - & - & - & - & - & - \\
\hline $\begin{array}{l}\text { Fusión intermedia } \\
\text { (<18-36 meses) }\end{array}$ & 4 & 2 & 66,67 & 33,33 & 2 & 6 & 25,00 & 75,00 & 3 & 3 & 50,00 & 50,00 \\
\hline $\begin{array}{l}\text { Fusión tardía } \\
\text { (<36-48 meses) }\end{array}$ & o & 2 & 0,00 & 100,00 & o & 5 & 0,00 & 100,00 & 4 & 2 & 66,67 & 33,33 \\
\hline Total & 4 & 7 & 36,36 & 63,64 & 2 & 11 & 15,38 & 84,62 & 7 & 5 & 58,33 & 41,67 \\
\hline
\end{tabular}

Tabla 4. Perfiles etarios de camélidos (Lama sp.). Referencias. F: Fusionado; NF: No fusionado; SUP: supervivencia; MOR: mortalidad.

taxón: 24,29\% ( $\mathrm{n}=34)$. En el C2, el 16,02\% ( $\mathrm{n}=29)$ de los especímenes de camélidos presentan algún tipo de marca de origen antrópico. Finalmente, el C3 presenta un $10,26 \%(n=8)$ de especímenes con ese tipo de marcas. En todos los casos predominan las marcas de corte (> 85\%) por sobre las de machacado, raspado (solo registradas en los Conjuntos 1 y 3) y percusión (solo identificadas en los Conjuntos 1 y 2).

Además, un total de cuatro especímenes óseos presentaron características que sugieren su formatización. Por un lado, para el C1 se registraron dos fragmentos óseos con evidencia de pulido en sus bordes y un fragmento de punta pulida. Por otro lado, para el C2 se registró un instrumento manufacturado sobre metapodio de camélido con punta roma y pulida y evidencia de desgaste en los cóndilos distales. Dado el soporte y la morfología del instrumento fue considerado como una wichuña, parte del toolkit empleado en la actividad textil (Izeta et al., 2013) (Figura 5).

Respecto al resto de los taxones (Rodentia, Caviidae, Chinchillidae, Dasypodidae, Aves), ninguno de sus especímenes presentó marcas de procesamiento aunque no se descarta su ingreso al basural producto del descarte posterior a su consumo. En este sentido, las placas dérmicas de los dasipódidos presentan termoalteración en su lado externo tanto en el $\mathrm{C} 1(\mathrm{n}=3)$ como en el C2 $(\mathrm{n}=1)$ lo que resulta un indicador indirecto de su consumo, considerando que una de las técnicas de cocción consiste en el asado del animal en su propio caparazón, apoyándolo sobre alguna fuente de calor (generalmente brasas).

También se llevó a cabo el análisis de las modificaciones óseas realizadas por agentes no antrópicos. En primer lugar, mientras que el C2 no registra marcas de carnívoros ni de roedores, los Conjuntos 1 y 3 poseen una frecuencia de marcas de estos agentes menor al $1 \%$ considerando el total del NISP para cada caso. En segundo lugar, se registraron los estadios de meteorización y en la Figura 6 se presentan los resultados obtenidos para el subconjunto Camelidae. Por un lado, los Conjuntos 1 y 2 exhiben una muy buena conservación dado que el 98,99\% y el 95\% del NISP respectivamente presentan estadios de meteorización entre 0 y 1 . Por otro lado, el C3 presenta especímenes con estadios de meteorización más avanzados: solo el 16,91\% presenta un estadio de 0 . Además, a diferencia de los otros dos conjuntos, el estadio más avanzado registrado es el 5. De este modo, el C3 debe haber estado expuesto a distintos agentes físico-químicos que alteraron los restos óseos de manera más intensa que en los otros dos conjuntos. Cabe destacar que anteriormente se presentó la correlación entre el \%MAU del esqueleto axial de Camelidae del C3 y los valores de densidad mineral ósea la cual resultó alta, positiva y significativa. Este dato sumado al grado de meteorización del subconjunto Camelidae del C3 refuerza la idea de que procesos de formación no antrópicos podrían haber alterado los restos óseos de dicho contexto con más intensidad que en los otros dos contextos. 

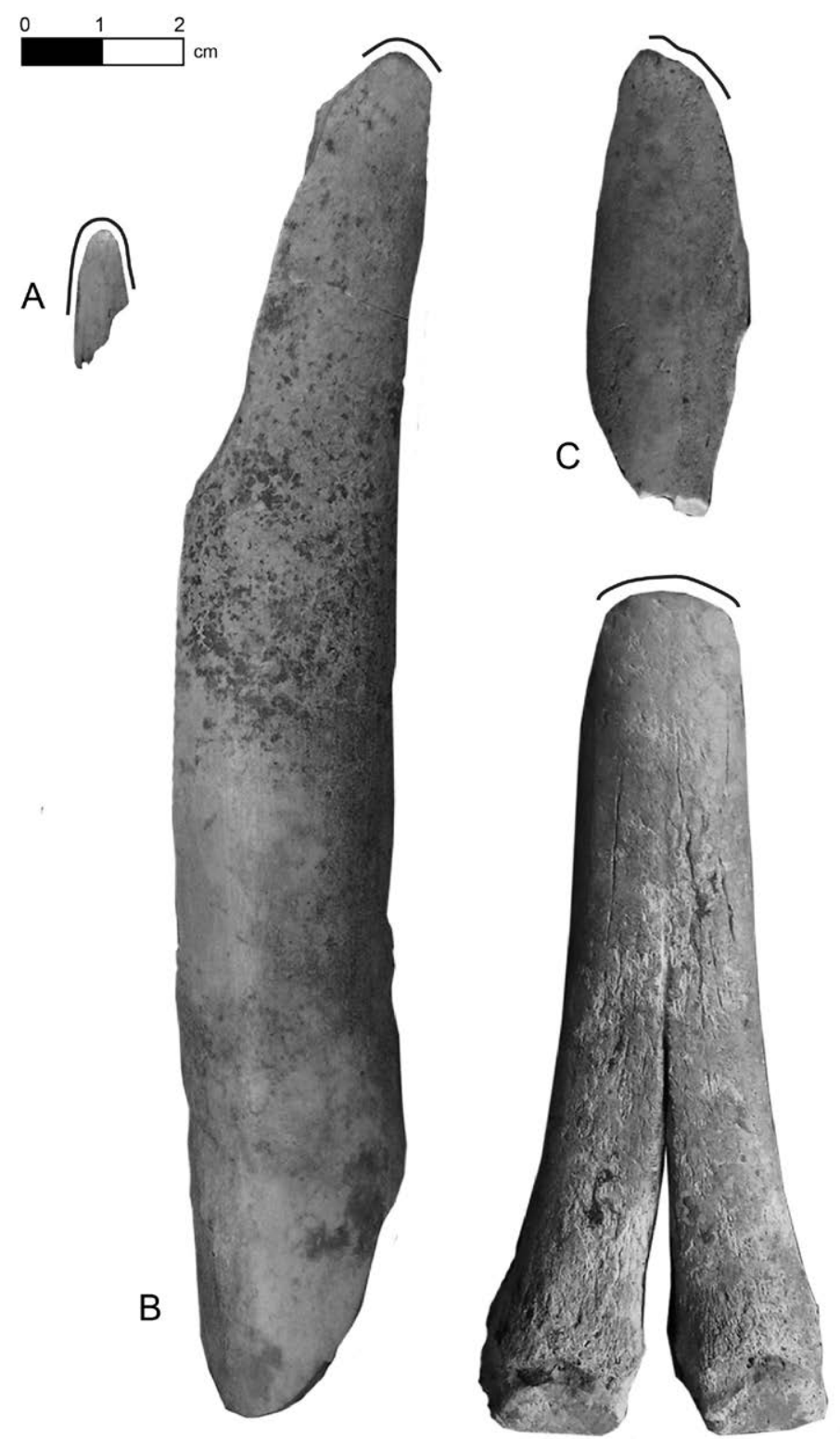

D

Figura 5. Restos óseos formatizados. Nota: las líneas marcan las zonas que presentan pulido. A, B y C provienen del Conjunto 1 y D del Conjunto 2.

\section{Discusión}

El material arqueofaunístico depositado en el basural de Palo Blanco permite discutir las estrategias vinculadas a la obtención, manejo, aprovechamiento y consumo de animales para los momentos más tempranos hasta ahora registrados en la aldea. Asimismo, la segmentación temporal del contexto de depositación permite comparar e identificar cambios y continuidades en dichas estrategias. De este modo, a continuación se discuten esos procesos, considerando cada uno de los bloques temporales delimitados: BT1 (400 a.C. - 1 d.C.), BT2 (1 d.C. - 400 d.C.) y BT3 (post 400 d.C.).

Antes de comenzar con la discusión es necesario destacar que la distribución dispersa de los NH de la aldea Palo Blanco puede ser atribuida al empleo de los grandes espacios entre las estructuras para el desarrollo de prácticas agrícolas. En este sentido, como se 


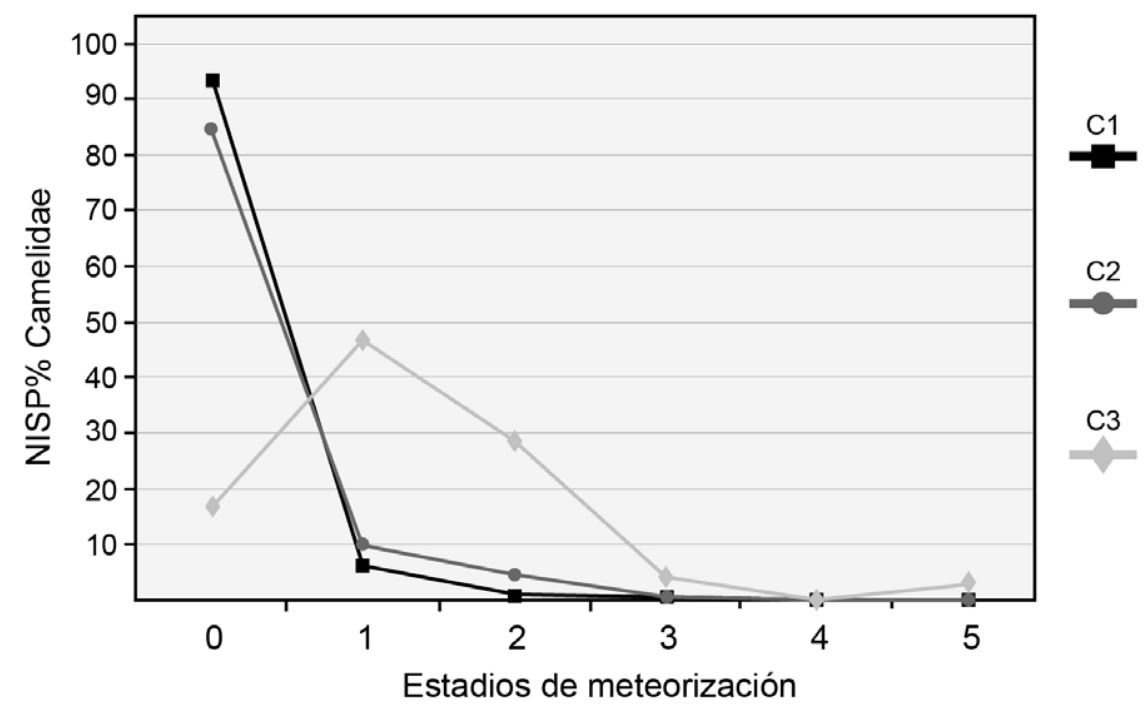

Figura 6. Estadios de meteorización de Camelidae.

destacó anteriormente, se recuperaron marlos de maíz quemados del basural, en su gran mayoría provenientes del BT1. Además, en todo el valle de Fiambalá se ha registrado evidencia arquitectónica (muros simples y "despedres") que dan cuenta del desarrollo de la actividad agrícola en el pasado. La combinación de esta información arqueológica y los datos geológicos de la región permitieron proponer que durante las primeras etapas del modo de vida productivo en el valle de Fiambalá los campos de cultivo se emplazaron dentro de la llanura aluvial, dado que se desarrolló una agricultura por inundación (Ratto, 2013). De este modo, la actividad agrícola habría sido el eje a partir de la cual las primeras sociedades productivas del valle de Fiambalá organizaron su asentamiento y subsistencia, tendencia observada para otros sectores de la región valliserrana (Izeta, 2008; Olivera, 2001). Por su parte, los animales también jugaron un rol importante en la subsistencia, creando así una economía productiva mixta (agricultura y pastoreo) complementada con actividades de caza y recolección.

Los camélidos se constituyen como los animales de mayor representación en toda la secuencia del basural de Palo Blanco y las llamas se encuentran presentes desde el inicio de la ocupación. Tanto para la región valliserrana como para la región puneña del NOA se ha propuesto que en los momentos más tempranos de las sociedades agropastoriles, los rebaños de llamas no habrían sido muy grandes debido al incipiente desarrollo del sistema ganadero (Belotti López de Medina, 2011; Olivera, 1997). En este sentido, y dada la temprana cronología de las capas inferiores del basural, consideramos que el tamaño de los rebaños habría sido pequeño también en Palo Blanco durante el BT1 y BT2, produciéndose un aumento con posterioridad a los 400 años d.C. (BT3) conforme eran controladas y perfeccionadas las técnicas de la actividad pastoril. A continuación, se discute la evidencia para sostener este postulado y para dar cuenta de los cambios y continuidades en las estrategias de subsistencia desarrolladas por los habitantes de la aldea de Palo Blanco a través del tiempo.

En primer lugar, si bien los camélidos predominan en los tres bloques temporales, la proporción respecto a otros taxones no es la misma. Así, para los momentos más tempranos (BT1) se destaca la importancia de los animales silvestres medianospequeños (roedores, dasipódidos y aves), conformando aproximadamente el $30 \% \mathrm{del}$ conjunto. En el BT2 la diversidad taxonómica se mantiene aunque la abundancia de la fauna menor disminuye para, finalmente, estar completamente ausente en el BT3. 
De este modo, los momentos más tempranos estarían marcados por una estrategia de diversificación en donde el pastoreo fue complementado con la caza de diversos animales silvestres. La reducción de la diversificación a través del tiempo marcaría un mayor control de las técnicas ganaderas y la consolidación del pastoreo como estrategia de aprovechamiento de animales. Respecto al tamaño de los rebaños, la estrategia de diversificación en la explotación de animales puede estar relacionada con la existencia de rebaños pequeños de llamas (Yacobaccio et al., 1997-1998).

Complementariamente al aumento de la importancia de los camélidos a través del tiempo, también ocurre un aumento de la diversidad de especies de esta familia. En este sentido, mientras que para el BT1 solo se registran camélidos domésticos, durante el BT2 se observan tanto llamas como posiblemente guanacos para finalmente en el BT3 registrarse llamas, posibles guanacos y vicuñas. Mientras que la caza de animales medianos-pequeños se reduce con el tiempo, la evidencia del basural muestra que para el BT2 y, principalmente, para el BT3 se registran camélidos silvestres: vicuñas y posiblemente guanacos. De este modo, las actividades cinegéticas como complemento de la actividad pastoril no desaparecen con el tiempo sino que se reorientarían hacia animales de mayor rinde económico como son los camélidos silvestres. Cabe señalar que la presencia de Vicugna vicugna en el BT3 sugiere la interacción de los habitantes de la aldea de Palo Blanco con la puna, hábitat natural de este camélido silvestre (Laker et al., 2006). Es una posibilidad que para momentos cercanos y posteriores a 400 d.C., la puna haya sido incorporada al movimiento estacional de los pastores de Palo Blanco (Miyano, 2016) dado los fechados del sitio El Zorro en la puna transicional de Chaschuil a 4.050 m s.n.m. (ca. 350-1050 d.C.) (Ratto, Carniglia y Coll, 2012), su régimen de ocupación temporal/estacional (Ratto, Kligmann y Russo, 2013) y la presencia de Vicugna vicugna en otro de los contextos de Palo Blanco, el NH6 (ca. 7001000 d.C.) (Miyano et al., 2015). Así, la evidencia de la incorporación de la puna en el movimiento estacional para momentos cercanos al 400 d.C. podría estar relacionada con el aumento del tamaño de los rebaños de la aldea y la necesidad incorporar más espacios con pasturas. La caza de vicuñas ocurrida en la puna habría evitado el sacrificio de llamas del rebaño para el consumo de carne en dicho piso altitudinal (Miyano, 2016). Además, no se debe descartar el aprovechamiento de la fibra de este camélido silvestre, considerando la existencia de evidencia indirecta que da cuenta del desarrollo de actividad textil en Palo Blanco (ver más adelante) y que la fibra de vicuña es la más fina dentro de la familia Camelidae (Quispe, Rodríguez, Iñiguez y Mueller, 2009).

En suma, durante los momentos más tempranos de la ocupación de Palo Blanco, la caza y fundamentalmente los recursos provenientes de las actividades agrícolas otorgaron los recursos que posibilitaron el sostenimiento de la aldea así como también el desarrollo del pastoreo y sus técnicas y el progresivo aumento del tamaño de los rebaños.

En segundo lugar, el análisis de los perfiles etarios permite, por un lado, abordar las estrategias adoptadas respecto a los camélidos domésticos y, por otro lado, otorgar evidencia acerca del tamaño de los rebaños. Para el BT1 y BT2 se observa un predominio de camélidos subadultos, tendencia que se revierte para el BT3. Si se analiza específicamente la estructura etaria es posible observar que en el BT1 y BT2 predominan los camélidos menores a los 12-36 meses, lo que sugiere la matanza de animales jóvenes. Esta matanza selectiva indicaría la obtención y aprovechamiento de productos primarios (carne, médula ósea, grasa) así como también apoyaría la idea de que el tamaño de los rebaños era pequeño durante los momentos más tempranos de la aldea, dado que el sacrificio de subadultos se llevaría a cabo para mantener a los rebaños en límites ecológicamente sustentables en contextos de sistemas ganaderos poco desarrollados durante los inicios de la vida agropastoril (Belotti López de Medina, 2011, 2015; Olivera, 1997). Asimismo, no se descarta la explotación de camélidos domésticos por sus productos secundarios (carga y/o fibra) durante este momento 
dado que, aunque escasa, existe evidencia de la conservación de animales de más de 36 meses de edad. Además, en el BT1 se registraron elementos óseos de grandes dimensiones que podrían pertenecer a llamas cargueras (Yacobaccio, 2010). Respecto a esto, Olivera (1997) sostiene que si bien el morfotipo de llama carguera se incrementa para el período Tardío-Inka, su uso en momentos tempranos está presente, aunque de manera restringida. En tanto, en el BT2 se recuperó una wichuña, instrumento asociado a la actividad textil. Este mismo tipo de instrumento fue registrado en sitios del sur de los valles Calchaquíes para momentos de las primeras sociedades agropastoriles y fueron vinculados con este tipo de actividad artesanal (Izeta et al., 2013). Complementariamente, en el BT2 hay presencia de llamas de tamaño pequeño las cuales podrían asignarse al morfotipo de llama lanuda (Yacobaccio, 2010).

El perfil etario observado en el BT3 es diferente a los del BT1 y BT2: se destaca un predominio de camélidos adultos, específicamente aquellos con una edad superior a los 36-48 meses. Esto sugiere la conservación de animales para el uso de sus productos secundarios (fibra y/o carga). En este sentido, los estudios osteométricos muestran que para este momento se registra el espécimen óseo de llama más grande de toda la secuencia, muy probablemente correspondiente a una llama carguera, así como también especímenes de llamas pequeñas asignables al morfotipo llama lanuda. Asimismo, no se descarta la explotación de camélidos por sus productos primarios considerando que los análisis de utilidad económica sugieren un aprovechamiento de aquellas partes del esqueleto apendicular con un alto contenido cárnico (ver más adelante) y que otros estudios arqueofaunísticos realizados en contextos posteriores al 400 d.C. en la aldea de Palo Blanco indicarían una matanza de individuos en su edad óptima como productores de carne (Miyano et al., 2015). Cabe recordar que el conjunto arqueofaunístico correspondiente a este bloque temporal del basural (C3) pudo haber estado sujeto a procesos de formación que alteraron significativamente su composición. En este sentido, existen estudios tafonómicos que indican que la densidad mineral ósea de los huesos de camélidos crías y juveniles es menor a la de los adultos y, por lo tanto, su comportamiento ante distintos procesos tafonómicos sería diferencial (Âlvarez, González, Massigoge, Kaufmann y Gutiérrez, 2010). De este modo, si mediaron procesos destructivos significativos, los huesos no fusionados estarían subrepresentados respecto a los fusionados y el perfil etario tendería a sobrerrepresentar la supervivencia. Sin embargo, en el C3 hay evidencia de huesos de baja densidad mineral ósea tales como metapodios sin fusionar (Álvarez et al., 2010), húmero y tibia proximal y segundas falanges (Elkin, 1995).

En suma, la evidencia discutida anteriormente indica el aprovechamiento de recursos primarios y secundarios de los camélidos domésticos en toda la secuencia del basural. Sin embargo, si tenemos en cuenta exclusivamente los perfiles etarios, en los momentos más tempranos la estrategia habría estado dirigida a la explotación de recursos primarios mientras que en momentos posteriores al 400 d.C. el aprovechamiento de los recursos secundarios aumentaría. En este sentido, un aumento progresivo del tamaño de los rebaños posibilitaría el desarrollo de morfotipos especializados para carga y/o fibra así como también la reserva de animales vivos para su uso nutricional.

4. En el caso de la vicuña, y asumiendo que su hábitat en el pasado coincide con el del presente, la distancia en línea recta desde Palo Blanco a la puna es de aproximadamente $60 \mathrm{~km}$.
Finalmente, las distintas partes esqueletarias que componen el esqueleto de un camélido se encuentran presentes en los tres bloques temporales. Resulta altamente probable que estos animales (al menos los domésticos) ingresaran completos a la aldea dado que el sacrificio se haría en sus inmediaciones. Este no sería el caso de los camélidos silvestres cuyas partes esqueletarias serían transportadas selectivamente dada la distancia entre el lugar de obtención y la aldea ${ }^{3}$. En este sentido, en los tres conjuntos predominan huesos del esqueleto apendicular que brindan mucha carne y también hay una buena representación del costillar y las cinturas. De este modo, los huesos del basural son el resultado de actividades de descarte de unidades anatómicas 
consumidas inmediatamente en la aldea y que poseen mucha carne y, en el caso de los huesos del esqueleto apendicular, médula ósea. Asimismo, para los momentos más tempranos (BT1) es posible que se haya realizado el secado de ciertas partes esqueletarias y su consumo haya sido diferido. Esta práctica podría relacionarse con la estrategia de diversificación de recursos postulada para estos momentos del inicio del desarrollo del pastoreo en la aldea: la carne seca y almacenada ampliaría aún más los recursos de origen animal disponibles para el consumo. Más allá de esto último, la representación de partes esqueletarias observadas en toda la secuencia del basural concuerda con actividades de descarte posterior al consumo de partes anatómicas con mucho contenido cárnico. En este sentido, los análisis de residuos orgánicos realizados en ollas de cocción procedentes del NH1, NH3, NH4 y NH6 (ca. 200-1000 d.C.) dan cuenta de la presencia de grasa de camélido lo que apoyaría el consumo de camélidos en la aldea (Lantos, Spangenberg, Giovannetti, Ratto y Maier, 2015).

\section{Consideraciones finales}

Los fechados radiocarbónicos realizados sobre materiales provenientes del basural de Palo Blanco le otorgaron mayor profundidad temporal a la aldea, aunque el estrato más profundo no cuenta aún con un núcleo habitacional para relacionarlo. En este sentido, el estudio de los conjuntos arqueofaunísticos del basural permitió abordar las estrategias de obtención, manejo, aprovechamiento y consumo de animales implementadas en la aldea para los momentos que coinciden con el inicio del modo de vida productivo en la región valliserrana. Asimismo, y dada la potencia del basural, fue posible evaluar cambios y continuidades en las estrategias vinculadas al uso de animales a lo largo del tiempo. Así, se discutió el desarrollo de la actividad pastoril en el tiempo, el rol de la fauna silvestre y las actividades cinegéticas, las modalidades de consumo de las distintas unidades anatómicas de los camélidos y los diversos usos tecnológicos que abarcan desde el uso de las llamas como animales de carga, el empleo de la fibra para la producción textil y el uso de los huesos para la manufactura de artefactos. De este modo, el análisis arqueofaunístico del basural nos otorgó información que permite acercarnos un poco más a la forma de vida de los aldeanos de Palo Blanco y su relación con los animales así como también a los inicios de la vida agropastoril del NOA en general.

\section{Agradecimientos}

El presente trabajo fue realizado en el marco de una Beca Interna Doctoral de CONICET y de los proyectos "Ciclos de vida y abandono en la aldea de Palo Blanco (siglos I y XI). Departamento Tinogasta, Catamarca” (UBACYT 20020130100071BA) y "Arqueología de la región de Fiambalá: cambios y continuidades en la interacción social entre las tierras bajas y altas (Siglos I a XVI)” (PICT-2012-0196). Agradezco a Norma Ratto por su ayuda constante así como también a los dos evaluadores anónimos cuyos comentarios permitieron mejorar el presente artículo. Finalmente, agradezco profundamente a Mariana De Nigris por sus enseñanzas a lo largo de todos estos años. Siempre será recordada por su alegría. 


\section{Referencias citadas}

» Albeck, M. E. (2000). La vida agraria en los Andes del Sur. En M. Tarragó (Ed.), Nueva Historia Argentina, volumen 1. Los Pueblos Originarios y la Conquista (pp. 187-228). Buenos Aires: Sudamericana.

» Álvarez, M., González, M., Massigoge, A., Kaufmann, C.y Gutiérrez, M. (2010). La densidad mineral ósea y la variabilidad ontogénica en guanaco (Lama guanicoe). Implicancias para la construcción de marcos de referencia en zooarqueología. En M. Gutiérrez, M., De Nigris, P. Fernández, M. Giardina, A. Gil, A. Izeta, G. Neme, y H. Yacobaccio (Eds.), Zooarqueología a Principios del Siglo XX: Aportes Teóricos, Metodológicos y Casos de Estudio (pp. 95-106). Buenos Aires: Libros del Espinillo.

»Behrensmeyer, A. (1978). Taphonomic and Ecologic Information from Bone Weathering. Paleobiology, 4, 150-162.

» Belotti López de Medina, C. R. (2011). Zooarqueología del sitio formativo Soria 2, valle de Yocavil (Catamarca), siglo I d.C. Revista del Museo de Antropología, 4, 3-16.

» Belotti López de Medina, C. R. (2015). Desigualdad e intensificación de la subsistencia en el valle de Yocavil (Catamarca y Tucumán, Argentina) entre los siglos I a.C. y XVI d. C. Relaciones de la Sociedad Argentina de Antropología, XL(1), 73-100.

»Binford, L. (1978). Nunamiut Ethnoarchaeology. Nueva York: Academic Press.

》 Binford, L. (1984). Faunal Remains from Klasies River Mouth. Orlando: Academic Press.

» Bonomo, N., Osella, A. y Ratto, N. (2010). Detecting and mapping buried buildings with GPR at an ancient village in Northwestern Argentina. Journal of Archaeological Science, 37, 3247-3255.

»Bronk Ramsey, C. (2009). Bayesian analysis of radiocarbon dates. Radiocarbon, 51(1), 337360.

»Broughton, J. (1994). Late Holocene resource intensification in the Sacramento Valley, California: The vertebrate evidence. Journal of Archaeological Science, 21, 501-514.

»Cabrera, A. y Willink, A. (1973). Biogeografía de América Latina. Washington: OEA.

»De Nigris, M. (2004). El Consumo en Grupos Cazadores Recolectores. Un Ejemplo Zooarqueológico de Patagonia Meridional. Buenos Aires: Sociedad Argentina de Antropología.

"De Nigris, M. y Mengoni Goñalons, G. (2005). The guanaco as a source of meat and fat in the Southern Andes. En J. Mulville y A. Outram (Eds.), The zooarchaeology of Fats, Oils and Dairying (pp. 6o-166). Oxford: Oxbow Books.

"Elkin, D. (1995). Volume Density of South American Camelid Skeletal Parts. International Journal of Osteoarchaeology, 5, 29-37.

"Grant, J. (2010). Aportes de distintas técnicas osteométricas para la identificación interespecífica de camélidos sudamericanos. En M. Gutiérrez, M., De Nigris, P. Fernández, M. Giardina, A. Gil, A. Izeta, G. Neme y H. Yacobaccio (Eds.), Zooarqueología a Principios del Siglo XX: Aportes Teóricos, Metodológicos y Casos de Estudio (pp. 17-28). Buenos Aires: Libros del Espinillo.

》 Grayson, D. (1984). Quantitative Zooarchaeology. Orlando: Academic Press.

" Grayson, D. (1991). Alpine faunas from the White Mountains, California: adaptive change in the late prehistoric Great Basin. Journal of Archaeological Science, 18, 483-506. 
"Izeta, A. (2007). Zooarqueología del Sur de los Valles Calchaquíes (Provincias de Catamarca y Tucumán, República Argentina): Análisis de Conjuntos Faunísticos del Primer Milenio A.D. Oxford: BAR International Series, Archaeopress.

》Izeta, A. (2008). Late Holocene camelid use tendencies in two different ecological zones of Northwestern Argentina. Quaternary International, 180, 135-144.

"Izeta, A. y Cortés, L. (2006). Southamerican Camelid palaeopathologies. Examples from Loma Alta (Catamarca, Argentina). International Journal of Osteoarchaeology, 16, 269-275.

" Izeta, A., Cattáneo, R., Scattolin, M. y Cortés, L. (2013). Changed into tools. Camelid bones from the Southern Calchaquíes Valleys (Formative Period, North-western Argentina). En A. Choyke y S. O'Connor (Eds.), From these Bare Bones: Raw Materials and the Study of Worked Osseous Objects (pp. 50-58). Oxford: Oxbow Books.

" Kent, J. (1982). The Domestication and exploitation of the South American camelids: methods of analysis and their application to circum-lacustrine archaeological sites in Bolivia and Peru. (Tesis Doctoral inédita), Washington University, USA.

" Klein, R. y Cruz-Uribe, K. (1984). The Analysis of Animal Bones from Archaeological Sites. Chicago: University of Chicago Press.

"Laguens, A. (2006). Continuidad y ruptura en procesos de diferenciación social en comunidades aldeanas del Valle de Ambato, Catamarca, Argentina (S. IV-X D.C.). Chungara. Revista de Antropología Chilena, 38(2), 211-222.

" Laker, J., Baldo, J., Arzamendia, Y. y Yacobaccio, H. (2006). La vicuña en los Andes. En B. Vilá (Ed.), Investigación, Conservación y Manejo de Vicuñas (pp. 37-50). Buenos Aires: Talleres Gráficos Leograf.

"Lantos, I., Spangenberg, J., Giovannetti, M., Ratto, N. y Maier, M. (2015). Maize consumption in pre-Hispanic south-central Andes: chemical and microscopic evidence from organic residues in archaeological pottery from western Tinogasta (Catamarca, Argentina). Journal of Archaeological Science, 55, 83-99.

"Lanzelotti, S. y Spano, R. (2015). La multitemporalidad del paisaje en la Mesada del Agua Salada (Catamarca, Argentina). Arqueología, 21(1), 47-71.

"López, G. (2003). Pastoreo y caza de camélidos en el Temprano de la Puna de Salta: Datos osteométricos del sitio Matancillas 2. Intersecciones en Antropología, $4,17-27$.

» Lyman, R. (1994). Vertebrate Taphonomy. Nueva York: Cambridge University Press.

" Lyman, R. (2008). Quantitative Paleozoology. Cambridge: Cambridge University Press.

» Martino, L., Bonomo, N., Lescano, E., Osella, A. y Ratto, N. (2006). Geoelectrical and GPR joint prospection in the ancient Palo Blanco archaeological site, NW Argentina. Geophysics, 71(6), 193-199.

" Meadow, R. (1987). Techniques for comparing bone measurement data from small samples. Trabajo presentado en Northeastern Faunal Analysis Conference, Storrs, USA.

" Mengoni Goñalons, G. (1991). La llama y sus productos primarios. Arqueología, 1, 179196.

》 Mengoni Goñalons, G. (1996). La domesticación de los camélidos sudamericanos y su anatomía económica. En D. Elkin, C. Madero, G. Mengoni Goñalons, D. Olivera, M. Reigadas y H. Yacobaccio (Eds.), Zooarqueología de Camélidos 2 (pp. 33-45). Buenos Aires: Grupo Zooarqueología de Camélidos.

" Mengoni Goñalons, G. (1999). Cazadores de Guanacos de la Estepa Patagónica. Buenos Aires: Sociedad Argentina de Antropología. 
» Mengoni Goñalons, G. (2006-2010). Zooarqueología en la práctica: algunos temas metodológicos. Xama, 19-23, 83-113.

» Mengoni Goñalons, G. (2008). Camelids in ancient Andean societies: A review of the zooarchaeological evidence. Quaternary International, 185, 59-68.

» Mengoni Goñalons, G. (2013). El aprovechamiento de la fauna en sociedades complejas: aspectos metodológicos y su aplicación en diferentes contextos arqueológicos del NOA. En V. Williams y M. B. Cremonte (Comps.), Al Borde del Imperio. Paisajes Sociales, Materialidad y Memoria en Áreas Periféricas del Noroeste Argentino (pp. 311-396). Buenos Aires: Sociedad Argentina de Antropología.

» Mengoni Goñalons, G. y Yacobaccio, H. (2006). The Domestication of South American Camelids. A View from the South-Central Andes. En M. Zeder, D. Bradley, E. Emshwiller y B. Smith (Eds.), Documenting Domestication. New Genetic and Archaeological Paradigms (pp. 228-244). Los Angeles: University of California Press.

» Miyano, J. P. (2016). Análisis zooarqueológico del sitio El Zorro (ca. 300-100o D.C., Puna Transicional de Chaschuil, Catamarca). En Actas del XIX Congreso Nacional de Arqueología Argentina (pp. 421-425). Tucumán: Universidad Nacional de Tucumán.

» Miyano, J. P., De Nigris, M. y Ratto, N. (2015). Zooarqueología de la aldea de Palo Blanco (Tinogasta, Catamarca). Revista del Museo de Antropología, 8(2), 7-20.

» Nastri, J., Coll Moritán, V. y Belotti López de Medina, C. R. (2012). El Intermedio Tardío en la Sierra del Cajón (provincia de Catamarca). Avance de las investigaciones en Morro del Fraile. Estudios Sociales del NOA, 12, 81-110.

» Olivera, D. (1997). La importancia del Recurso Camelidae en la Puna de Atacama entre los 10.000 y 500 años A.P. Estudios Atacameños, 14, 29-41.

» Olivera, D. (2001). Sociedades agropastoriles tempranas: el Formativo Inferior del Noroeste Argentino. En E. Berberián y A. Nielsen (Eds.), Historia Argentina Prehispánica I (pp. 83-126). Córdoba: Editorial Brujas.

»Olivera, D. (2012). El Formativo en los Andes del Sur: la incorporación de la opción productiva. En M. de Haro, A. Rocchietti, M. Runcio, O. Hernández de Lara y M. Fernández (Eds.), Interculturalidad y Ciencias: Experiencias desde América Latina (pp. 1549). Buenos Aires: Centro de Investigaciones Precolombinas.

» Osella A., Bonomo, N. y Ratto, N. (2009). Prospección geofísica en la localidad arqueológica de Palo Blanco y alrededores (Departamento Tinogasta, Catamarca). En N. Ratto (Comp.), Entrelazando Ciencias: Sociedad y Ambientes Antes de la Conquista Española (pp. 67-98). Buenos Aires: EUDEBA.

» Quispe, E., Rodríguez, T., Iñiguez, L. y Muelle, J. P. (2009). Producción de fibra de alpaca, llama, vicuña y guanaco en Sudamérica. Animal Genetic Resources Information, 45, 1-14.

» Ratto, N. y Basile, M. (2010). La localidad arqueológica de Palo Blanco (Dpto. Tinogasta, Catamarca): nuevas evidencias. En R. Bárcena y H. Chiavazza (Eds.), Actas del XVII Congreso Nacional de Arqueología Argentina (Tomo IV) (pp. 1707-1712). Mendoza: Universidad Nacional de Cuyo.

» Ratto, N., Carniglia, D. y Coll, L. (2012). Ocupación del área de “Los Seismiles” desde tiempo formativos a recientes (Departamento Tinogasta, Catamarca): nuevas tendencias. Relaciones de la Sociedad Argentina de Antropología, XXXVII(1), 207-216.

» Ratto, N., Kligmann, D. y Russo, N. (2013). Geoarqueología de sitios de altura (4000-500o msnm) del oeste tinogasteño (Catamarca). En N. Ratto (Comp.), Delineando prácticas de la gente del pasado. Los Procesos Socio-Históricos del Eeste Tinogasteño (Catamarca) (pp. 357-377). Buenos Aires: Sociedad Argentina de Antropología. 
» Ratto, N., Montero, M., Hongn, F. y Valero Garcés, B. (2013). La historia ambiental de las sociedades productivas del oeste tinogasteño (Catamarca, siglos I a XVI). En Ratto, N. (Comp.), Delineando Prácticas de la Gente del Pasado. Los procesos socio-históricos del oeste tinogasteño (Catamarca) (pp. 45-68). Buenos Aires: Sociedad Argentina de Antropología.

» Ratto, N., Basile, M., Feely, A., Lantos, I., Coll, L., Carniglia, D. y Miyano, J. P. (2015). La gente y sus prácticas en las tierras bajas y altas del oeste tinogasteño en los siglos I a XIII D.C. (Catamarca, Argentina). En A. Korstanje, M. Lazzari, M. Basile, F. Bugliani, V. Lema, L. Pereyra Domingorena y M. Quesada (Eds.), Crónicas Materiales Precolombinas. Arqueología de los Primeros Poblados del Noroeste Argentino (pp. 215-145). Buenos Aires: Sociedad Argentina de Antropología.

»Scattolin. M. (2007). Santa María antes del año mil. Fechas y materiales para una historia cultural. En V. Williams, B. Ventura, A. Callegari y H. Yacobaccio (Eds.), Sociedades Precolombinas Surandinas: Temporalidad, Interacción y Dinámica cultural del NOA en el Ámbito de los Andes Centro-Sur (pp. 203-219). Buenos Aires: Artes Gráficas Buschi.

»Scattolin, M., Pereyra Domingorena, L., Cortés, L., Bugliani, F., Calo, C., Izeta, A. y Lazzari, M. (2007). Cardonal: una aldea formativa entre los territorios de valles y Puna. Cuadernos de la Facultad de Humanidades y Ciencias Sociales - Universidad Nacional de Jujuy, 32, 211-225.

»Sempé, M. (1976). Contribución a la arqueología del valle de Abaucán. (Tesis Doctoral inédita), Universidad Nacional de La Plata, Argentina.

» Vervoorst, F. (1951). Resultados de un viaje a la Cuenca de Laguna Verde (Tinogasta Catamarca). III. Observaciones sobre la vegetación entre Tinogasta y la cuenca. En Actas de la XV Semana de Geografía (pp. 61-67). Mendoza: Universidad Nacional de Cuyo.

»Von den Driesch, A. (1976). A Guide to the Measurement of Animal Bones from Archaeological Sites. Bulletin I. Cambridge: Peabody Museum of Archaeology and Ethnology.

»Yacobaccio, H. (2010). Osteometría de llamas (Lama glama L.) y sus consecuencias arqueológicas. En M. Gutiérrez, M. De Nigris, P. Fernández, M. Giardina, A. Gil, A. Izeta, G. Neme y H. Yacobaccio (Eds.), Zooarqueología a Principios del Siglo XX: Aportes Teóricos, Metodológicos y Casos de Estudio (pp. 65-75). Buenos Aires: Libros del Espinillo.

» Yacobaccio, H., Madero, C., Malmierca, M. y Reigadas, M. (1997-1998). Caza, domesticación y pastoreo de camélidos en la Puna Argentina. Relaciones de la Sociedad Argentina de Antropología, XXII-XXIII, 389-418. 